\title{
Introduction to Bosonization
}

\author{
E. Miranda \\ Instituto de Física Gleb Wataghin, Unicamp, \\ Caixa Postal 6165, 13083-970, Campinas, SP, Brazil \\ emiranda@ifi.unicamp.br
}

Received on 27 August, 2002

\begin{abstract}
This is a pedagogical introduction to the general technique of bosonization of one-dimensional systems starting from scratch and assuming very little besides basic quantum mechanics and second quantization. The formalism is developed in a self-contained fashion and applied to the spinless and spin- $\frac{1}{2}$ Luttinger models, working out both single and two particle correlation functions. The implications of these results for the specific cases of the (anisotropic) Heisenberg and the Hubbard models are discussed. Although everything in these notes can be found in the published literature, detailed and explicit calculations of most of the results are given, which may prove useful to beginning graduate students or researchers in this area.
\end{abstract}

\section{Introduction}

These notes formed the basis of a series of lectures given at the Brazilian Statistical Mechanics School, which took place from February 18 to 29, 2002, at the Universidade de São Paulo in São Carlos. While writing them, I had in mind a beginning graduate student in physics, already familiar with basic Quantum Mechanics, including the formalism of second quantization, but not with very much more. I follow through the mathematical details necessary to establish the bosonization technique of one-dimensional systems, which is by now a rigorous and mature method that underlies much of our understanding of these systems. It has found many applications in real quasi-one-dimensional systems such as quantum wires [1], carbon nanotubes [2] and edge states of the quantum Hall effect [3]. For the sake of motivation, I focus on two models: the Hubbard model of spin- $\frac{1}{2}$ fermions and the anisotropic (XXZ) Heisenberg spin- $\frac{1}{2}$ model. I should stress that all the material covered in these lectures can be found in one way or another in the published literature, so there is no claim of originality. However, the detail and care with which some calculations are done may be useful for the uninitiated, who are the main targets of these notes.

The topic of bosonization is covered in many review articles. Some of then are $[4,5,6,7,8]$. Some of the original articles are $[9,10,11,12,13,14,15,16]$. I have drawn extensively from Haldane [15], von Delft and Schoeller [8], Voit[7] and Affleck [6].

These notes are organized as follows. Section II introduces the two basic models. The fundamental tools of bosonization are developed in Sections III to XIII. Section XIV focuses on the basic interacting model solved by bosonization, the Luttinger model. This is then applied to the XXZ model in Section XV. Section XVI is devoted to the important Luttinger liquid conjecture by Haldane. The case of spin- $-\frac{1}{2}$ fermions is studied in Section XVII. We end with a brief discussion of gaps and the sine-Gordon theory in Section XVIII.

\section{The Hubbard and the Heisenberg models}

Our aim will be to study strongly correlated systems in one spatial dimension. These are typically systems of interacting electrons but we will be interested in spin systems as well.

The prototypical interacting electron system is the Hubbard model. This is a lattice model whose Hamiltonian in one dimension is

$$
H_{H u b}=-t \sum_{j \sigma}\left(c_{j \sigma}^{\dagger} c_{j+1 \sigma}+\text { h.c. }\right)+U \sum_{j} c_{j \uparrow}^{\dagger} c_{j \uparrow} c_{j \downarrow}^{\dagger} c_{j \downarrow} .
$$

The first term describes the hopping process, in which an electron can move from one site to the next with amplitude $t$ while preserving its spin projection $\sigma$ (taken arbitrarily along the $z$-axis). The second term describes the local Coulomb repulsion $(U>0)$ between opposite spin electrons residing on the same site. This is the so-called Hubbard $U$ interaction term, named after one of the first people to work on this model in a series of classic papers [17, 18, 19]. This is one of the simplest interacting fermionic models one can write and has been extensively studied.

The $c_{j \sigma}$ operators are the usual annihilation operators with anti-commutation relations 


$$
\begin{aligned}
\left\{c_{j \sigma}, c_{j^{\prime} \sigma^{\prime}}\right\} & =\left\{c_{j \sigma}^{\dagger}, c_{j^{\prime} \sigma^{\prime}}^{\dagger}\right\}=0, \\
\left\{c_{j \sigma}, c_{j^{\prime} \sigma^{\prime}}^{\dagger}\right\} & =\delta_{j, j^{\prime}} \delta_{\sigma, \sigma^{\prime}} .
\end{aligned}
$$

Another important model is the spin $-1 / 2 \mathrm{XXZ}$ model,

$$
H_{X X Z}=J \sum_{j}\left(S_{j}^{x} S_{j+1}^{x}+S_{j}^{y} S_{j+1}^{y}+\Delta S_{j}^{z} S_{j+1}^{z}\right) \text {. }
$$

Here, $S_{j}^{a}$ are spin- $\frac{1}{2}$ operators with commutation relations

$$
\left[S_{j}^{a}, S_{j^{\prime}}^{b}\right]=i \delta_{j, j^{\prime}} \varepsilon^{a b c} S_{j}^{c} \quad a=x, y, z \text { or equivalently } 1,2,3,
$$

and $\sum_{a}\left(S_{j}^{a}\right)^{2}=\frac{3}{4}=\frac{1}{2}\left(\frac{1}{2}+1\right)$. The symbol $\varepsilon^{a b c}$ is the totally anti-symmetric Levi-Civita tensor

$$
\varepsilon^{a b c}=\left\{\begin{array}{cc}
0 & \text { if there are repeated indices among }(\mathrm{a}, \mathrm{b}, \mathrm{c}) \\
1 & \text { if }(\mathrm{a}, \mathrm{b}, \mathrm{c}) \text { is an even permutation of }(1,2,3) \\
-1 & \text { if }(\mathrm{a}, \mathrm{b}, \mathrm{c}) \text { is an odd permutation of }(1,2,3)
\end{array} .\right.
$$

$J$ is the exchange coupling and $\Delta$ the anisotropy parameter. A special important case of (4) is at $\Delta=1$, the so-called isotropic Heisenberg model

$$
H_{H e i s}=J \sum_{j} \mathbf{S}_{j} \cdot \mathbf{S}_{j+1} \cdot
$$

Both models (1) and (4) can be solved exactly in one dimension (and only in one dimension) by means of the celebrated Bethe Ansatz [20, 21]. However, though the Bethe Ansatz can give the spectrum of eigenvalues and eigenvectors (plus a bit more), there is still a lot of important information that it cannot give, such as correlation functions.

The technique of bosonization, specially suited for one spatial dimension, is a powerful field-theoretical tool that enables one to calculate correlation functions. In fact, it gives us a very great deal of insight into the physics of onedimensional systems by classifying them into "universality classes" and by characterizing their spectrum of low-lying excitations.

Getting ahead of ourselves, it consists of a systematic mapping of a fermionic system (states, operators, Hamiltonians, etc.) into an auxiliary bosonic one. It turns out that the bosonic language is often more suited for the understanding of the physics of the system, sometimes even allowing for its exact solution, as we will see.

We will embark on this construction taking the Hubbard model as a guide and it will become clear how it can be generalized to other models.

Let us first look at the non-interacting limit $(U=0)$. In this case, the Hamiltonian can be easily diagonalized by means of Fourier transformation. Define (we work with the lattice spacing $a=1$ )

$$
c_{k \sigma}^{\dagger}=\sum_{j=1}^{L} \frac{e^{i k j}}{\sqrt{L}} c_{j \sigma}^{\dagger} \Leftrightarrow c_{k \sigma}=\sum_{j=1}^{L} \frac{e^{-i k j}}{\sqrt{L}} c_{j \sigma}
$$

and the inverse transformation

$$
c_{j \sigma}^{\dagger}=\sum_{k \in B Z} \frac{e^{-i k j}}{\sqrt{L}} c_{k \sigma}^{\dagger} \Leftrightarrow c_{j \sigma}=\sum_{k \in B Z} \frac{e^{i k j}}{\sqrt{L}} c_{k \sigma} .
$$

Note that we have "put the system in a box (ring)", which is short for working on a finite lattice of $L$ sites, with periodic boundary conditions

$$
c_{j+L, \sigma}^{\dagger}=\sum_{k \in B Z} \frac{e^{-i k j} e^{-i k L}}{\sqrt{L}} c_{k \sigma}^{\dagger}=c_{j \sigma}^{\dagger} .
$$

The last equality follows if

$$
e^{-i k L}=1 \Rightarrow k=\frac{2 \pi}{L} n,
$$

where

$$
n=0, \pm 1, \pm 2, \ldots, \pm\left(\frac{L}{2}-1\right), \frac{L}{2} .
$$

We take even values of $L$ even for simplicity. Higher values of $n$ are redundant since, if $k=\frac{2 \pi}{L}\left(\frac{L}{2}+1\right)$, then

$e^{i k j}=e^{i \frac{2 \pi}{L}\left(\frac{L}{2}+1\right) j}=e^{i \frac{2 \pi}{L}\left(L-\frac{L}{2}+1\right) j}=e^{-i \frac{2 \pi}{L}\left(\frac{L}{2}-1\right) j}=e^{i k^{\prime} j}$,

where $k^{\prime}=\frac{2 \pi}{L}\left[-\left(\frac{L}{2}-1\right)\right]$ and $k$ is completely equivalent to $k^{\prime}$. The set (12) is called the first Brillouin zone (hence the $k \in B Z$ ). Note that $\frac{L}{2}$ and $-\frac{L}{2}$ are also equivalent (by a similar argument) and we keep only $\frac{L}{2}$. The inverse transformation is now easily proved

$$
\begin{aligned}
& c_{j \sigma}^{\dagger}=\sum_{k \in B Z} \frac{e^{-i k j}}{\sqrt{L}} c_{k \sigma}^{\dagger}=\sum_{k \in B Z} \frac{e^{-i k j}}{\sqrt{L}} \sum_{l=1}^{L} \frac{e^{i k l}}{\sqrt{L}} c_{l \sigma}^{\dagger} \\
& =\frac{1}{L} \sum_{l=1}^{L} \sum_{k \in B Z} e^{i k(l-j)} c_{l \sigma}^{\dagger}=c_{j \sigma}^{\dagger}
\end{aligned}
$$

where we used (see Appendix A.1 for the proof)

$$
\sum_{k \in B Z} e^{i k j}=L \delta_{j, 0}
$$


It is important to have the anti-commutation relations in $k$ space

$$
\left\{c_{k \sigma}, c_{k^{\prime} \sigma^{\prime}}\right\}=\left\{c_{k \sigma}^{\dagger}, c_{k^{\prime} \sigma^{\prime}}^{\dagger}\right\}=0,
$$

$$
\left\{c_{k \sigma}, c_{k^{\prime} \sigma^{\prime}}^{\dagger}\right\}=\delta_{k, k^{\prime}} \delta_{\sigma, \sigma^{\prime}}
$$

which are also easily proved.

Taking these expressions into the non-interacting Hamiltonian, we have

$$
\begin{aligned}
& H_{0}=-t \sum_{j \sigma}\left(c_{j \sigma}^{\dagger} c_{j+1 \sigma}+\text { h.c. }\right)=-t \sum_{j \sigma}\left[\sum_{k \in B Z} \frac{e^{-i k j}}{\sqrt{L}} c_{k \sigma}^{\dagger} \sum_{p \in B Z} \frac{e^{i p(j+1)}}{\sqrt{L}} c_{p \sigma}+\right.\text { h.c. } \\
& =-\frac{t}{L} \sum_{k, p \in B Z} \sum_{j \sigma} e^{i j(p-k)} e^{i p} c_{k \sigma}^{\dagger} c_{p \sigma}+\text { h.c. }=-\mathrm{t} \sum_{\mathrm{k} \in \mathrm{BZ}} \mathrm{e}^{\mathrm{ik}} c_{\mathrm{k} \sigma}^{\dagger} c_{\mathrm{k} \sigma}+\text { h.c. } \\
& =-2 t \sum_{k \in B Z} \cos (k) c_{k \sigma}^{\dagger} c_{k \sigma}=\sum_{k \in B Z} \varepsilon(k) c_{k \sigma}^{\dagger} c_{k \sigma},
\end{aligned}
$$

where we have used Appendix A.1. The Hamiltonian is now diagonal in the $k$-basis and is easily solved,

$$
c_{k \sigma}^{\dagger} c_{k \sigma} \equiv n_{k \sigma}=0 \text { or } 1 \text { (for each } \sigma \text { and } \mathrm{k} \text { ). }
$$

The ground state for $N$ electrons corresponds to filling up all the states, from the lowest energy up, until the $N$ lowestenergy orbitals are filled up (with due care of spin degeneracy). The highest occupied level is the Fermi level, its energy the Fermi energy $E_{F}$ and its wave-vector the Fermi wave-vector $k_{F}$ (see Fig. 1). The relation between $N$ and $k_{F}$ is

$$
\begin{aligned}
N & =L \int_{-k_{F}}^{k_{F}} \frac{d k}{2 \pi} \times 2=\frac{2 k_{F} L}{\pi} \\
\Rightarrow n & \equiv \frac{N}{L}=\frac{2 k_{F}}{\pi} .
\end{aligned}
$$

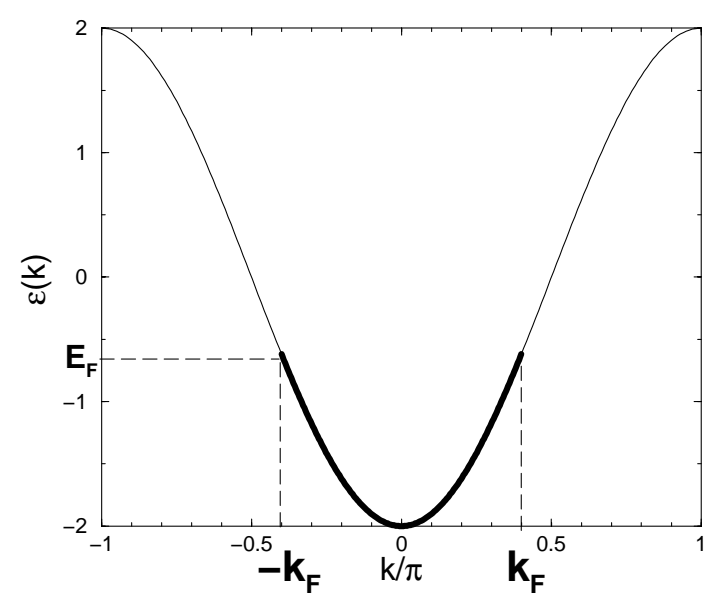

Figure 1. The non-interacting Fermi sea.

The extra factor of 2 comes from spin degeneracy. We used the fact that, in the thermodynamic limit $(L \rightarrow \infty)$

$$
\sum_{k} f(k)=\sum_{n=-L / 2+1}^{L / 2} f\left(\frac{2 \pi n}{L}\right) \approx \frac{L}{2 \pi} \int_{-\pi}^{\pi} d k f(k),
$$

where $f(k)$ is a general function of $k$. In the example above, $f(k)=\theta\left(k_{F}-|k|\right)$, where $\theta(x)$ is the usual Heaviside theta-(step-)function.

\section{Linearized spectrum}

Let us now look at the effect of interactions. If $U \ll t$ (perturbative region), it is natural to assume that only low energy states will be much affected. This is reasonable within second order perturbation theory, where

$$
\begin{aligned}
\Delta E_{0}^{(2)} & \sim \sum_{n \neq 0} \frac{\left\langle 0\left|H_{U}\right| n\right\rangle\left\langle n\left|H_{U}\right| 0\right\rangle}{E_{0}-E_{n}}, \\
\Delta|0\rangle^{(2)} & \sim \sum_{n \neq 0} \frac{\left\langle n\left|H_{U}\right| 0\right\rangle}{E_{0}-E_{n}}|n\rangle
\end{aligned}
$$

where $H_{U}$ is the interaction part of Eq. (1). It is clear that the denominator suppresses corrections coming from higher energies. We thus can reasonably focus on the low-energy subspace.

Looking at the dispersion relation, it is reasonable to linearize the spectrum around the two Fermi points (see Fig. 2), if we are going to be concerned mostly with weakly excited states. We will choose the zero energy so that $E_{F}=0$. Note that the reduction of the Fermi sphere to two disconnected "Fermi points" is a feature specific to one dimension and central to the upcoming developments.

Now, since the two dispersions, cosine and linear, differ only at higher excitation energies, we will simply replace one by the other. But remember that we are effectively restricted to low energies if we want to say something about 
the Hubbard model. Now we have two branches of excitations in the V-shaped spectrum of Fig. 2. Finally, we can extend each branch so that $\mathrm{k}$ runs from $-\infty$ to $\infty$, effectively introducing an infinite number of states in each branch, the so-called "positron" states, by analogy with the Dirac spectrum in one dimension. We therefore abandon for a moment the Hubbard model and focus on the "linear dispersion model" of Fig. 3. Because we have an infinite number of states in each branch, we will have to introduce certain careful "cut-off" procedures to correctly define the theory. We will call the branches

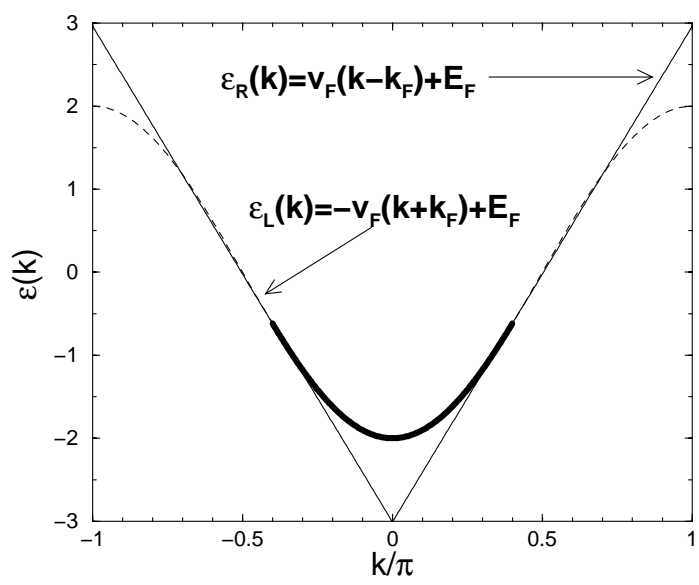

Figure 2. Linearization of the spectrum.

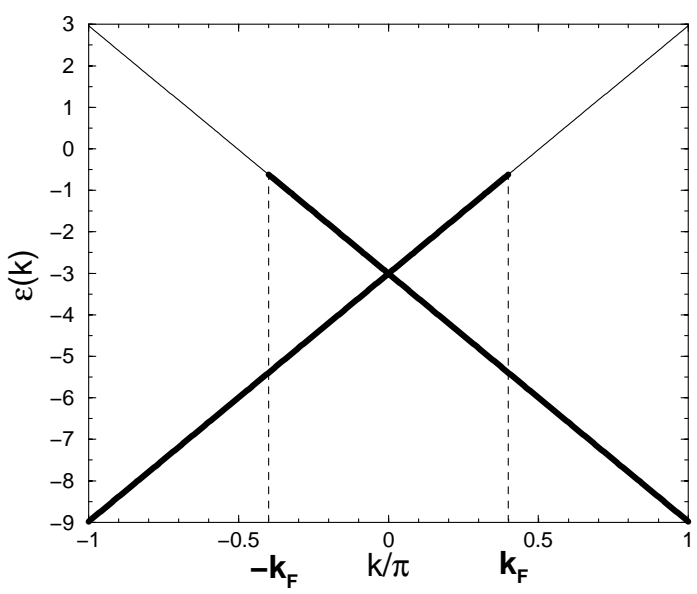

Figure 3. The linear dispersion model.

$\varepsilon(k)=v_{F}\left(k-k_{F}\right) \Longrightarrow$ Right moving branch,(22)
$\varepsilon(k)=-v_{F}\left(k+k_{F}\right) \Longrightarrow$ Left moving branch,

according to the signs of the velocities. The relation between this spectrum and the lattice one is given by

$$
v_{F}=\left.\frac{\partial \varepsilon(k)}{\partial k}\right|_{k=k_{F}}=2 t \sin \left(k_{F}\right) .
$$

Let us for now focus on the right movers. We still want to work "in a box" with periodic boundary conditions so

$$
k=\frac{2 \pi n}{L}
$$

but now

$$
n=0, \pm 1, \pm 2, \ldots
$$

since the spectrum is not bounded. This is equivalent to working in the continuum limit, where the lattice spacing $a \rightarrow 0$ and the Brillouin zone $\left(-\frac{\pi}{a}, \frac{\pi}{a}\right) \rightarrow(-\infty, \infty)$. The creation and annihilation operators still satisfy Eqs. (15). We now define field operators $\psi(x)$, operator-valued functions of the continuous variable $x \in\left[-\frac{L}{2}, \frac{L}{2}\right]$, which are the continuum limit analogs of $c_{j \sigma}$ (we forget about spin for a moment)

$$
\begin{aligned}
\psi(x) & \equiv \frac{1}{\sqrt{L}} \sum_{k=-\infty}^{+\infty} e^{i k x} c_{k}, \\
\psi^{\dagger}(x) & \equiv \frac{1}{\sqrt{L}} \sum_{k=-\infty}^{+\infty} e^{-i k x} c_{k}^{\dagger}
\end{aligned}
$$

It is better to think of this as a definition. We will worry about how to relate $\psi(x)$ to $c_{j}$ more precisely later. From now on $\sum_{k}=\sum_{k=-\infty}^{+\infty}$. Note that $\psi(x)$ is periodic with period $L$

$$
\psi(x+L)=\psi(x)
$$

It follows that

$$
\{\psi(x), \psi(y)\}=\left\{\psi^{\dagger}(x), \psi^{\dagger}(y)\right\}=0,
$$

and

$$
\begin{aligned}
& \left\{\psi(x), \psi^{\dagger}(y)\right\}=\frac{1}{L} \sum_{k, p} e^{i k x} e^{-i p y}\left\{c_{k}, c_{p}^{\dagger}\right\} \\
& =\frac{1}{L} \sum_{k} e^{i k(x-y)}=\frac{1}{L} \sum_{n=-\infty}^{+\infty} \delta\left(\frac{x-y}{L}-n\right) \\
& =\sum_{n=-\infty}^{+\infty} \delta(x-y-n L)
\end{aligned}
$$

where we used Eq. (362) of Appendix A.2. If $(x, y) \in$ $\left(-\frac{L}{2}, \frac{L}{2}\right) \Rightarrow\left\{\psi(x), \psi^{\dagger}(y)\right\}=\delta(x-y)$ which is the analogue of $\left\{c_{j}, c_{j^{\prime}}^{\dagger}\right\}=\delta_{j, j^{\prime}}$ in the continuum. Furthermore

$$
c_{k}=\frac{1}{\sqrt{L}} \int_{-\frac{L}{2}}^{\frac{L}{2}} d x e^{-i k x} \psi(x)
$$

is the inverse transformation. 


\section{Hilbert space}

In field theory, where we have an infinite number of degrees of freedom, it is important to be specific about the Hilbert space we are working with, as this is not always obvious from the Hamiltonian. Let us do that for our linearized branch.

First, let's imagine that $k_{F}=0$, for simplicity. Then we start from a vacuum state $|0\rangle_{0}$, which is the "Dirac sea" of an infinite number of fermions occupying all states with $k \in(-\infty, 0]$ or

$$
k=\frac{2 \pi n}{L}, \quad n=0,-1,-2, \ldots,
$$

as depicted in Fig. 4

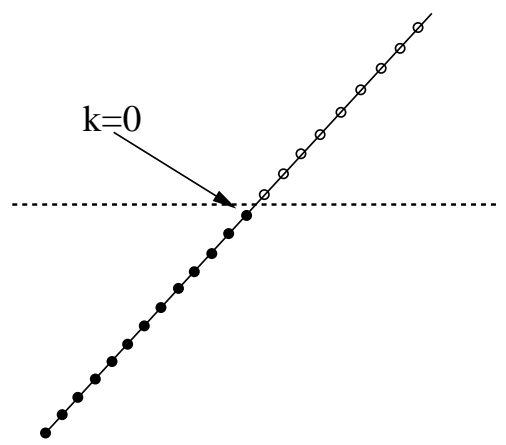

Figure 4 . The vacuum state $|0\rangle_{0}$ of the right moving branch.

Thus,

$$
\begin{aligned}
& c_{k}|0\rangle_{0}=0, \quad k>0, \\
& c_{k}^{\dagger}|0\rangle_{0}=0, \quad k \leq 0 .
\end{aligned}
$$

The Hilbert space $\mathcal{H}$ is spanned by all the states that can be generated by acting with a finite number of $c_{k}$ or $c_{k}^{\dagger}$ on $|0\rangle_{0}$, see Fig. 5. It is useful to classify these states according to the total number of fermions. Since this is an infinite number, we use instead the difference between the total number of fermions of the state and that of $|0\rangle_{0}$, which is a finite number. In other words, we use the number operator

$$
\hat{N}=\sum_{k}\left[c_{k}^{\dagger} c_{k}-\left\langle c_{k}^{\dagger} c_{k}\right\rangle_{0}\right]
$$

where $\langle-\rangle_{0}=0\langle 0|-| 0\rangle_{0}$. It is also customary to define the operation of normal-ordering a string of creation and annihilation operators $A B C D \ldots$, usually denoted by : $A B C D \ldots$. . It amounts to moving all operators that destroy the vacuum (Eq. (34)) to the right by doing transpositions and multiplying by -1 each time. For example, if $k_{1} \leq 0, k_{2}>0, k_{3}>0, k_{4} \leq 0$, then

$$
: c_{k_{1}}^{\dagger} c_{k_{2}}^{\dagger} c_{k_{3}} c_{k_{4}}:=-c_{k_{2}}^{\dagger} c_{k_{4}} c_{k_{1}}^{\dagger} c_{k_{3}} .
$$

It is equivalent to the above subtraction of the vacuum expectation value when there are only two operators involved

$$
\hat{N}=\sum_{k}: c_{k}^{\dagger} c_{k}:
$$

Besides, the two operations are equivalent when taking averages in the vacuum (though this is sometimes omitted), since

$$
\langle: A B C D \ldots:\rangle_{0}=0 \text {. }
$$

Thus, we can group all states of $\mathcal{H}$ according to $N$, eigenvalues of $\hat{N}$. It is clear that $N \in \mathbb{Z}$.. For fixed $N$, the lowest-energy state, the one with no particle-hole excitations, will be called the $N$-particle ground state (see Fig. 5(a) for an example)

$$
\begin{aligned}
c_{N}^{\dagger} c_{N-1}^{\dagger} \cdots c_{1}^{\dagger}|0\rangle_{0} & \equiv|N\rangle_{0}(N>0), \\
c_{N+1} c_{N+2} \cdots c_{-1} c_{0}|0\rangle_{0} & \equiv|N\rangle_{0}(N<0) .
\end{aligned}
$$

The Hilbert space with fixed number of particles $\mathcal{H}_{\mathcal{N}}$ is spanned by all numbers of particle-hole excitations on the corresponding $N$-particle ground state (see Fig. 5(b) for an example). It follows that

$$
\mathcal{H}=\mathcal{H}_{0} \oplus \mathcal{H}_{1} \oplus \mathcal{H}_{2} \oplus \cdots
$$

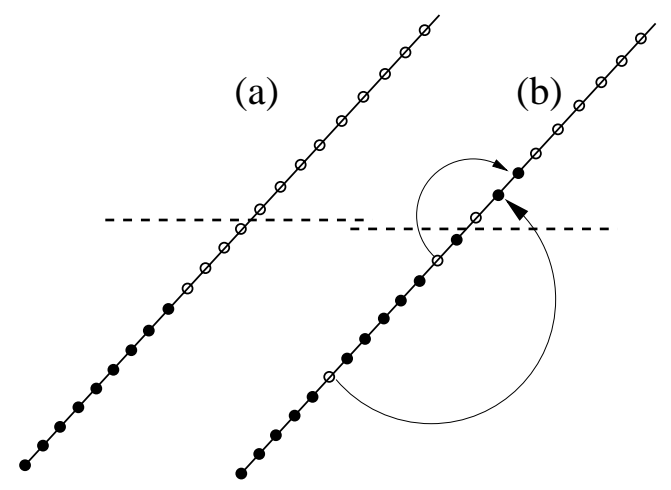

Figure 5. Examples of states that generate the Hilbert space $\mathcal{H}$. (a) The four highest energy particles of $|0\rangle_{0}$ have been removed. This is the -4 -particle ground state $|-4\rangle_{0}$; (b) Two particle-hole excitations have been created on $|0\rangle_{0}$.

\section{Density operators}

We now define an important linear combination of operators that create particle-hole excitations, the density fluctuation operators, or simply density operators, for $q \neq 0$ only

$$
\rho(q) \equiv \sum_{k} c_{k+q}^{\dagger} c_{k}
$$

Note that $\rho(-q)=\rho^{\dagger}(q)$. The reason why they are called density (fluctuation) operators becomes clear from 


$$
\begin{aligned}
& : \psi^{\dagger}(x) \psi(x):=\frac{1}{L} \sum_{k p} e^{i(k-p) x}: c_{p}^{\dagger} c_{k}:=\frac{1}{L} \sum_{k q} e^{-i q x}: c_{k+q}^{\dagger} c_{k}: \\
& =\frac{1}{L} \sum_{q \neq 0} e^{-i q x} \sum_{k}: c_{k+q}^{\dagger} c_{k}:+\frac{1}{L} \sum_{k}: c_{k}^{\dagger} c_{k}:=\frac{\hat{N}}{L}+\frac{1}{L} \sum_{q \neq 0} e^{-i q x} \rho(q) .
\end{aligned}
$$

In the last step, we removed the normal-ordering sign in the first term, because it is redundant when the two fermion operators have different $k$-indices. Let's calculate its commutators (we make use of $[A, B C]=\{A, B\} C-B\{A, C\}$ )

$$
\begin{aligned}
& {[\rho(p), \rho(q)]=\sum_{k k^{\prime}}\left[c_{k^{\prime}+p}^{\dagger} c_{k^{\prime}}, c_{k+q}^{\dagger} c_{k}\right]=\sum_{k k^{\prime}}\left\{c_{k^{\prime}+p}^{\dagger}\left[c_{k^{\prime}}, c_{k+q}^{\dagger} c_{k}\right]+\left[c_{k^{\prime}+p}^{\dagger}, c_{k+q}^{\dagger} c_{k}\right] c_{k^{\prime}}\right\}} \\
& =\sum_{k k^{\prime}}\left\{c_{k^{\prime}+p}^{\dagger} \delta_{k^{\prime}, k+q} c_{k}-c_{k+q}^{\dagger} c_{k^{\prime}} \delta_{k^{\prime}+p, k}\right\}=\sum_{k}\left[c_{k+p+q}^{\dagger} c_{k}-c_{k+q}^{\dagger} c_{k-p}\right] .
\end{aligned}
$$

If $p \neq-q$,

$$
[\rho(p), \rho(q)]=\rho(p+q)-\rho(p+q)=0 .
$$

If, however, $p=-q$,

$$
[\rho(p), \rho(-p)]=\sum_{k}\left[: c_{k}^{\dagger} c_{k}:+\left\langle c_{k}^{\dagger} c_{k}\right\rangle_{0}-: c_{k-p}^{\dagger} c_{k-p}:-\left\langle c_{k-p}^{\dagger} c_{k-p}\right\rangle_{0}\right] .
$$

We can now make the shift $k-p \rightarrow k$ within the normal ordering sign because it does not introduce infinite quantities. The two terms cancel. We are left with

$$
\begin{aligned}
{[\rho(p), \rho(-p)] } & =\sum_{k}\left[\left\langle c_{k}^{\dagger} c_{k}\right\rangle_{0}-\left\langle c_{k-p}^{\dagger} c_{k-p}\right\rangle_{0}\right] \\
& =-\frac{L}{2 \pi} p .
\end{aligned}
$$

Note the importance of the presence of the infinite Dirac sea. Thus

$$
[\rho(p), \rho(q)]=-\frac{L p}{2 \pi} \delta_{p,-q}
$$

This is an example of a current algebra. It almost looks like a bosonic commutation relation. We can make it precisely that by defining

$$
\begin{aligned}
b_{q} & =\sqrt{\frac{2 \pi}{L q}} \rho(-q) \quad(q>0), \\
b_{q}^{\dagger} & =\sqrt{\frac{2 \pi}{L q}} \rho(q) \quad(q>0)
\end{aligned}
$$

such that

$$
\begin{aligned}
& {\left[b_{q}, b_{q^{\prime}}\right]=\left[b_{q}^{\dagger}, b_{q^{\prime}}^{\dagger}\right]=0} \\
& {\left[b_{q}, b_{q^{\prime}}^{\dagger}\right]=\delta_{q, q^{\prime}}}
\end{aligned}
$$

These are bona fide bosonic creation and annihilation operators. We can write

$$
\rho(q)= \begin{cases}\sqrt{\frac{L q}{2 \pi}} b_{q}^{\dagger} & q>0 \\ \sqrt{\frac{L|q|}{2 \pi}} b_{-q} & q<0\end{cases}
$$

and

$$
\begin{aligned}
: \psi^{\dagger}(x) \psi(x): & =\frac{\hat{N}}{L}+\frac{1}{L} \sum_{q>0}\left[e^{-i q x} \rho(q)+e^{i q x} \rho(-q)\right] \\
& =\frac{\hat{N}}{L}+\frac{1}{\sqrt{2 \pi L}} \sum_{q>0} \sqrt{q}\left[e^{i q x} b_{q}+e^{-i q x} b_{q}^{\dagger}\right]
\end{aligned}
$$


It is quite easy to show that

$$
\left[b_{q}, \hat{N}\right]=\left[b_{q}^{\dagger}, \hat{N}\right]=0
$$

Finally, note that

$$
b_{q}|N\rangle_{0}=0, \quad \forall q, N .
$$

Physically, this means that the $N$-particle ground state contains no particle-hole excitations.

Normal ordering bosons (also denoted by : $A B C \ldots$ :) means transposing $b_{q}$ 's to the right and $b_{q}^{\dagger}$ 's to the left, with no accompanying sign. Likewise, it is equivalent to subtracting the vacuum expectation values (when taking averages with respect to the vacuum state) and for two operators

$$
: A B:=A B-\langle A B\rangle_{0} .
$$

\section{Completeness of the bosonic rep- resentation}

There is an important theorem, due to Haldane [15], that shows that the $N$-particle Hilbert space $\mathcal{H}_{\mathcal{N}}$, spanned by all possible particle-hole excitation of $|N\rangle_{0}$, is also spanned by applying $b_{q}^{\dagger}$ 's on $|N\rangle_{0}$ any number of times

$$
|N\rangle \in \mathcal{H}_{N} \Rightarrow|N\rangle=f\left[\left\{b_{q}^{\dagger}\right\}\right]|N\rangle_{0},
$$

where we denoted a general ket in $\mathcal{H}_{\mathcal{N}}$ by $|N\rangle$ and $f\left[\left\{b_{q}^{\dagger}\right\}\right]$ is an general function of the bosonic creation operators. Therefore, we have a faithful representation of $\mathcal{H}_{\mathcal{N}}$ in terms of bosons.

\section{Klein factors}

Hilbert spaces with different numbers of particles, however, cannot be connected with $b_{q}^{\dagger}$ 's, $b_{q}$ 's or $\hat{N}$. But, fermionic creation and annihilation operators do just that. Therefore, to complete the bosonic prescription we need to define new operators, called Klein factors, that change $N$ by one. We will call it $F$, with the following defining properties

(i) $\left[F, b_{q}^{\dagger}\right]=\left[F, b_{q}\right]=\left[F^{\dagger}, b_{q}^{\dagger}\right]=\left[F^{\dagger}, b_{q}\right]=0$,
$(i i) \quad F^{\dagger}|N\rangle_{0}=|N+1\rangle_{0}$,

(iii) $\quad F|N\rangle_{0}=|N-1\rangle_{0}$.
It follows that, for a general $N$-particle state $|N\rangle=$ $f\left[\left\{b_{q}^{\dagger}\right\}\right]|N\rangle_{0}$

$$
\begin{gathered}
F^{\dagger}|N\rangle=f\left[\left\{b_{q}^{\dagger}\right\}\right] F^{\dagger}|N\rangle_{0}=f\left[\left\{b_{q}^{\dagger}\right\}\right]|N+1\rangle_{0}, \\
F|N\rangle=f\left[\left\{b_{q}^{\dagger}\right\}\right] F|N\rangle_{0}=f\left[\left\{b_{q}^{\dagger}\right\}\right]|N-1\rangle_{0} .
\end{gathered}
$$

In other words $F^{\dagger}|N\rangle(F|N\rangle)$ contains the same particlehole excitations as $|N\rangle$, but created on a different, $(N+1)$ $((N-1)-)$ particle ground state. It is clear from its definition that $F$ is unitary $F^{-1}=F^{\dagger}$. Also,

$$
\begin{aligned}
{[F, \hat{N}] } & =F, \\
{\left[F^{\dagger}, \hat{N}\right] } & =-F^{\dagger} .
\end{aligned}
$$

We can now, with the help of $F, F^{\dagger}$, and $b_{q}^{\dagger}$ 's, acting on $|0\rangle_{0}$ generate the whole Hilbert space $\mathcal{H}$.

\section{Fermionic creation and annihilation operators}

We now establish one of the most important building blocks of the bosonization dictionary: the expression of the fermionic annihilation operator $\psi(x)$ in terms of bosons, $\hat{N}$, and Klein factors. For that, we first derive the commutators of $\psi(x)$ with the bosons

$$
\begin{aligned}
{\left[b_{q}, \psi(x)\right] } & =\sqrt{\frac{2 \pi}{L q}} \frac{1}{\sqrt{L}} \sum_{k p} e^{i p x}\left[c_{k-q}^{\dagger} c_{k}, c_{p}\right] \\
& =-\sqrt{\frac{2 \pi}{L q}} \frac{e^{-i q x}}{\sqrt{L}} \sum_{k} e^{i k x} c_{k} \\
& =-\sqrt{\frac{2 \pi}{L q}} e^{-i q x} \psi(x), \\
{\left[b_{q}^{\dagger}, \psi(x)\right] } & =\sqrt{\frac{2 \pi}{L q}} \frac{1}{\sqrt{L}} \sum_{k p} e^{i p x}\left[c_{k+q}^{\dagger} c_{k}, c_{p}\right] \\
& =-\sqrt{\frac{2 \pi}{L q}} e^{i q x} \psi(x) .
\end{aligned}
$$

Applying Eq. (65) on the $N$-particle ground state $|N\rangle_{0}$

$$
\left[b_{q}, \psi(x)\right]|N\rangle_{0}=b_{q} \psi(x)|N\rangle_{0}=-\sqrt{\frac{2 \pi}{L q}} e^{-i q x} \psi(x)|N\rangle_{0} \equiv \alpha_{q}(x) \psi(x)|N\rangle_{0}
$$

Thus, $\psi(x)|N\rangle_{0}$ is an eigenstate of $b_{q}$, with eigenvalue $\alpha_{q}(x)$, for any $q>0$. Eigenstates of bosonic annihilation operators are called coherent states (See Appendix B, for a brief discussion). Since $\psi(x)|N\rangle_{0} \in \mathcal{H}_{N-1}$

$$
\psi(x)|N\rangle_{0} \propto \exp \left[\sum_{q>0} \alpha_{q}(x) b_{q}^{\dagger}\right]|N-1\rangle_{0},
$$


or

$$
\psi(x)|N\rangle_{0}=\Lambda(x) \exp \left[\sum_{q>0} \alpha_{q}(x) b_{q}^{\dagger}\right] F|N\rangle_{0},
$$

where $\Lambda(x)$ is a c-number. It can be determined by

${ }_{0}\left\langle N\left|F^{\dagger} \psi(x)\right| N\right\rangle_{0}=\Lambda(x)_{0}\left\langle N\left|\exp \left[\sum_{q>0} \alpha_{q}(x) b_{q}^{\dagger}\right]\right| N\right\rangle_{0}$,

and since $\exp \left[\sum_{q>0} \alpha_{q}^{*}(x) b_{q}\right]|N\rangle_{0}=|N\rangle_{0}$, the righthand side is $\Lambda(x)$. The left-hand side can be calculated

$$
{ }_{0}\left\langle N-1\left|\frac{1}{\sqrt{L}} \sum_{k=-\infty}^{+\infty} e^{i k x} c_{k}\right| N\right\rangle_{0}=\frac{1}{\sqrt{L}} e^{i \frac{2 \pi N x}{L}}=\Lambda(x),
$$

since only the value of $k=\frac{2 \pi N}{L}$ is left of the sum. Thus,

$$
\psi(x)|N\rangle_{0}=\frac{F}{\sqrt{L}} e^{i \frac{2 \pi \hat{N} x}{L}} \exp \left[\sum_{q>0} \alpha_{q}(x) b_{q}^{\dagger}\right]|N\rangle_{0} .
$$

We would like to generalize this to any ket $|N\rangle \in \mathcal{H}_{N}$. For that, the result of Section VI comes in handy

$$
|N\rangle=f\left[\left\{b_{q}^{\dagger}\right\}\right]|N\rangle_{0}
$$

We have

$$
\psi(x)|N\rangle=\psi(x) f\left[\left\{b_{q}^{\dagger}\right\}\right]|N\rangle_{0} .
$$

Using Eq. (66),

$$
\begin{aligned}
\psi(x) b_{q}^{\dagger} & =\left[b_{q}^{\dagger}-\alpha_{q}^{*}(x)\right] \psi(x) \\
\Rightarrow \psi(x)\left(b_{q}^{\dagger}\right)^{n} & =\left[b_{q}^{\dagger}-\alpha_{q}^{*}(x)\right]^{n} \psi(x) \\
\Rightarrow \psi(x) f\left[\left\{b_{q}^{\dagger}\right\}\right] & =f\left[\left\{b_{q}^{\dagger}-\alpha_{q}^{*}(x)\right\}\right] \psi(x) .
\end{aligned}
$$

Then,

$$
\begin{aligned}
\psi(x)|N\rangle & =f\left[\left\{b_{q}^{\dagger}-\alpha_{q}^{*}(x)\right\}\right] \psi(x)|N\rangle_{0} \\
& =f\left[\left\{b_{q}^{\dagger}-\alpha_{q}^{*}(x)\right\}\right] \frac{F}{\sqrt{L}} e^{i \frac{2 \pi \hat{N} x}{L}} \exp \left[\sum_{q>0} \alpha_{q}(x) b_{q}^{\dagger}\right]|N\rangle_{0} \\
& =\frac{F}{\sqrt{L}} e^{i \frac{2 \pi \hat{N} x}{L}} \exp \left[\sum_{q>0} \alpha_{q}(x) b_{q}^{\dagger}\right] f\left[\left\{b_{q}^{\dagger}-\alpha_{q}^{*}(x)\right\}\right]|N\rangle_{0}
\end{aligned}
$$

Making use of the identity 1 in Appendix C,

$$
\begin{aligned}
\exp \left[-\sum_{q>0} \alpha_{q}^{*}(x) b_{q}\right] b_{q}^{\dagger} \exp \left[\sum_{q>0} \alpha_{q}^{*}(x) b_{q}\right] & =b_{q}^{\dagger}-\alpha_{q}^{*}(x) \\
\Rightarrow \exp \left[-\sum_{q>0} \alpha_{q}^{*}(x) b_{q}\right] f\left[b_{q}^{\dagger}\right] \exp \left[\sum_{q>0} \alpha_{q}^{*}(x) b_{q}\right] & =f\left[\left\{b_{q}^{\dagger}-\alpha_{q}^{*}(x)\right\}\right] .
\end{aligned}
$$

Finally,

$$
\begin{aligned}
& \psi(x)|N\rangle=\frac{F}{\sqrt{L}} e^{i \frac{2 \pi \hat{N} x}{L}} \exp \left[\sum_{q>0} \alpha_{q}(x) b_{q}^{\dagger}\right] \exp \left[-\sum_{q>0} \alpha_{q}^{*}(x) b_{q}\right] f\left[b_{q}^{\dagger}\right] \exp \left[\sum_{q>0} \alpha_{q}^{*}(x) b_{q}\right]|N\rangle_{0} \\
& \psi(x)|N\rangle=\frac{F}{\sqrt{L}} e^{i \frac{2 \pi \hat{N} x}{L}} \exp \left[\sum_{q>0} \alpha_{q}(x) b_{q}^{\dagger}\right] \exp \left[-\sum_{q>0} \alpha_{q}^{*}(x) b_{q}\right]|N\rangle
\end{aligned}
$$

This is one of the most important results of bosonization: the expression of the fermionic annihilation operator in terms of the bosons, $F$ and $\hat{N}$. It is called the Mattis-Mandelstam formula, after some of its discoverers [11, 13, 22, 23]. 


\section{Bosonic field operators}

It will prove useful to define bosonic field operators by

$$
\begin{aligned}
\varphi(x) & =-\frac{i}{\sqrt{2 \pi}} \sum_{q>0} \alpha_{q}^{*}(x) e^{-\alpha q / 2} b_{q}=\frac{i}{\sqrt{L}} \sum_{q>0} \frac{e^{i q x}}{\sqrt{q}} e^{-\alpha q / 2} b_{q} \\
\varphi^{\dagger}(x) & =\frac{i}{\sqrt{2 \pi}} \sum_{q>0} \alpha_{q}(x) e^{-\alpha q / 2} b_{q}^{\dagger}=-\frac{i}{\sqrt{L}} \sum_{q>0} \frac{e^{-i q x}}{\sqrt{q}} e^{-\alpha q / 2} b_{q}^{\dagger} \\
\phi(x) & =\varphi(x)+\varphi^{\dagger}(x)=-\frac{i}{\sqrt{2 \pi}} \sum_{q>0} e^{-\alpha q / 2}\left[\alpha_{q}^{*}(x) b_{q}-\alpha_{q}(x) b_{q}^{\dagger}\right] \\
& =\frac{i}{\sqrt{L}} \sum_{q>0} \frac{1}{\sqrt{q}} e^{-\alpha q / 2}\left[e^{i q x} b_{q}-e^{-i q x} b_{q}^{\dagger}\right] .
\end{aligned}
$$

The "converging factor" $e^{-\alpha q / 2}$ is important in defining a proper bosonic theory in $1 \mathrm{D}$. These equations should always be viewed as having $e^{-\alpha q / 2}$ to ensure convergence at intermediate steps, but final results should be written taking $\alpha \rightarrow 0^{+}$.

A useful result is

$$
\begin{aligned}
\partial_{x} \phi & =-\frac{1}{\sqrt{L}} \sum_{q>0} \sqrt{q} e^{-\alpha q / 2}\left[e^{i q x} b_{q}+e^{-i q x} b_{q}^{\dagger}\right] \\
\Rightarrow: \psi^{\dagger}(x) \psi(x): & =\frac{\hat{N}}{L}-\frac{1}{\sqrt{2 \pi}} \partial_{x} \phi .
\end{aligned}
$$

We leave as exercises the following relations, where the $\approx$ sign means we have taken the limit $|x-y| \ll L$.

$$
\begin{aligned}
& {[\varphi(x), \varphi(y)]=\left[\varphi^{\dagger}(x), \varphi^{\dagger}(y)\right]=0 .} \\
& {\left[\varphi(x), \varphi^{\dagger}(y)\right]=-\frac{1}{2 \pi} \ln \left\{1-\exp \left[\frac{2 \pi i}{L}(x-y+i \alpha)\right]\right\} \approx-\frac{1}{2 \pi} \ln \left\{-\frac{2 \pi i}{L}(x-y+i \alpha)\right\}-\frac{i}{2 L}(x-y+i \alpha) \text {. }} \\
& {[\phi(x), \phi(y)]=-\frac{1}{2 \pi} \ln \left\{\frac{1-\exp \left[\frac{2 \pi i}{L}(x-y+i \alpha)\right]}{1-\exp \left[-\frac{2 \pi i}{L}(x-y-i \alpha)\right]}\right\} \approx \frac{i}{\pi} \arctan \left(\frac{x-y}{\alpha}\right)-\frac{i}{L}(x-y)} \\
& \stackrel{\alpha \rightarrow 0}{\longrightarrow} \frac{i}{2} \operatorname{sgn}(x-y)-\frac{i}{L}(x-y) \text {. } \\
& {\left[\varphi(x), \partial_{y} \varphi^{\dagger}(y)\right]=\frac{i}{L} \frac{1}{1-\exp \left[-\frac{2 \pi i}{L}(x-y+i \alpha)\right]} \approx \frac{1}{2 \pi} \frac{1}{x-y+i \alpha}+\frac{i}{2 L}} \\
& {\left[\partial_{x} \varphi(x), \varphi^{\dagger}(y)\right]=-\frac{i}{L} \frac{1}{1-\exp \left[-\frac{2 \pi i}{L}(x-y+i \alpha)\right]} \approx-\frac{1}{2 \pi} \frac{1}{x-y+i \alpha}-\frac{i}{2 L} \text {. }} \\
& {\left[\phi(x), \partial_{y} \phi(y)\right]=\frac{i}{L}\left\{\frac{1}{1-\exp \left[-\frac{2 \pi i}{L}(x-y+i \alpha)\right]}+\frac{1}{1-\exp \left[\frac{2 \pi i}{L}(x-y-i \alpha)\right]}\right\} \approx-\frac{i}{\pi} \frac{\alpha}{(x-y)^{2}+\alpha^{2}}+\frac{i}{L}} \\
& \stackrel{\alpha \rightarrow 0}{\longrightarrow}-i \delta(x-y)+\frac{i}{L} \text {. }
\end{aligned}
$$

Prove also that, as $\alpha \rightarrow 0^{+}$and for any $|x-y|$

$$
\left[\phi(x), \partial_{y} \phi(y)\right]=\frac{i}{L}-i \sum_{n=-\infty}^{\infty} \delta(x-y-n L) .
$$

Using identity 2 of Appendix C, we can write Eq. (81) as

$$
\begin{aligned}
& \psi(x)=\frac{F}{\sqrt{L}} e^{i \frac{2 \pi \hat{N} x}{L}} \exp \left[-i \sqrt{2 \pi} \varphi^{\dagger}(x)\right] \exp [-i \sqrt{2 \pi} \varphi(x)] \\
& \psi(x)=\frac{F}{\sqrt{2 \pi \alpha}} e^{i \frac{2 \pi \hat{N} x}{L}} \exp [-i \sqrt{2 \pi} \phi(x)] .
\end{aligned}
$$


Note that Eq. (93) is normal-ordered, whereas Eq. (94) is not. As a result, a diverging factor $\frac{1}{\sqrt{2 \pi \alpha}}$ appears in the latter.

As another exercise, prove that

$$
\psi^{\dagger}(x+a) \psi(x) \stackrel{\alpha \rightarrow 0}{\longrightarrow} \frac{i}{2 \pi a}+\frac{\hat{N}}{L}-\frac{1}{\sqrt{2 \pi}} \partial_{x} \phi
$$

to first sub-leading order in $a / L$. If

$: \psi^{\dagger}(x+a) \psi(x):=\psi^{\dagger}(x+a) \psi(x)-\left\langle\psi^{\dagger}(x+a) \psi(x)\right\rangle_{0}$,

then we recover Eq. (85).

\section{Hamiltonian with a linear disper- sion}

We saw that, for low energies, one can linearize the dispersion. For right-movers we get $\varepsilon(k)=v_{F} k$ so that

$$
H_{0}=v_{F} \sum_{k} k: c_{k}^{\dagger} c_{k}: \text {. }
$$

The normal ordering sign ensures the subtraction of the diverging ground state energy. We would like to find an expression for $H_{0}$ in terms of bosons. There is a quick and physically transparent way of arriving at the final result [15]. First, we look at the $N$-particle ground state $|N\rangle_{0}$. It is clear that it is an eigenvector of $H_{0}$ with eigenvalue $E_{N}^{(0)}$

$$
\begin{aligned}
\frac{E_{N}^{(0)}}{v_{F}} & = \begin{cases}\sum_{k=2 \pi / L}^{2 \pi N / L} k=\frac{2 \pi}{L} \sum_{n=1}^{N} n=\frac{\pi}{L} N(N+1) & \text { for } \mathrm{N}>0 \\
0 & \text { for } \mathrm{N}=0 \\
\sum_{k=2 \pi / L}^{2 \pi N / L}-k=-\frac{2 \pi}{L} \sum_{n=1}^{N} n=\frac{\pi}{L} N(N+1) & \text { for } \mathrm{N}<0\end{cases} \\
& =\frac{\pi}{L} N(N+1) .
\end{aligned}
$$

It is clear that $H_{0}$ conserves the number of particles, so we can try to find its action within a given $\mathcal{H}_{N}$. From the commutators of $H_{0}$ with the bosons

$$
\left[\frac{H_{0}}{v_{F}}, b_{q}^{\dagger}\right]=\sum_{k p} k \sqrt{\frac{2 \pi}{L q}}\left[: c_{k}^{\dagger} c_{k}:, c_{p+q}^{\dagger} c_{p}\right] .
$$

However,

$$
\begin{aligned}
& {\left[: c_{k}^{\dagger} c_{k}:, c_{p+q}^{\dagger} c_{p}\right]=\left[c_{k}^{\dagger} c_{k}, c_{p+q}^{\dagger} c_{p}\right]} \\
& =c_{k}^{\dagger}\left[c_{k}, c_{p+q}^{\dagger} c_{p}\right]+\left[c_{k}^{\dagger}, c_{p+q}^{\dagger} c_{p}\right] c_{k}=\delta_{k, p+q} c_{k}^{\dagger} c_{p}-\delta_{k, p} c_{p+q}^{\dagger} c_{k} .
\end{aligned}
$$

Therefore,

$$
\begin{aligned}
& {\left[\frac{H_{0}}{v_{F}}, b_{q}^{\dagger}\right]=\sum_{k p} k \sqrt{\frac{2 \pi}{L q}}\left(\delta_{k, p+q} c_{k}^{\dagger} c_{p}-\delta_{k, p} c_{p+q}^{\dagger} c_{k}\right)} \\
& =\sum_{p}(p+q) \sqrt{\frac{2 \pi}{L q}} c_{p+q}^{\dagger} c_{p}-\sum_{p} p \sqrt{\frac{2 \pi}{L q}} c_{p+q}^{\dagger} c_{p}=q \sum_{p} \sqrt{\frac{2 \pi}{L q}} c_{p+q}^{\dagger} c_{p}=q b_{q}^{\dagger}
\end{aligned}
$$

If $\left|N, E_{N}\right\rangle$ is an eigenstate of $H_{0}$ with eigenenergy $E_{N}$, then from Eq. (101), $b_{q}^{\dagger}\left|N, E_{N}\right\rangle$ is also an eigenstate with

$$
H_{0} b_{q}^{\dagger}\left|N, E_{N}\right\rangle=\left(E_{N}+v_{F} q\right) b_{q}^{\dagger}\left|N, E_{N}\right\rangle
$$

In other words, the bosonic quanta added by $b_{q}^{\dagger}$ have energies $v_{F} q$. Now, by acting with $b_{q}^{\dagger}$ 's on $|N\rangle_{0}$ we can generate the $N$-particle Hilbert space $\mathcal{H}_{N}$ as we saw

$$
|N\rangle=f\left[\left\{b_{q}^{\dagger}\right\}\right]|N\rangle_{0} .
$$

Thus, the only possible form for $H_{0}$ is

$$
H_{0}=v_{F} \sum_{q>0} q b_{q}^{\dagger} b_{q}+\frac{\pi}{L} v_{F} \hat{N}(\hat{N}+1) .
$$

The term in $\pi v_{F} \hat{N} / L$ is often dropped in the thermodynamic limit. In position space, we can also write, using Eq. (27), (28), and (84)

$$
H_{0}=v_{F} \int_{-L / 2}^{L / 2} d x: \psi^{\dagger}(x)\left(-i \partial_{x}\right) \psi(x):
$$




$$
H_{0}=\frac{v_{F}}{2} \int_{-L / 2}^{L / 2} d x:\left(\partial_{x} \phi\right)^{2}:+\frac{\pi}{L} v_{F} \hat{N}(\hat{N}+1)
$$

which we leave as an exercise. Prove also that Eq. (106) can be obtained directly from Eq. (105) by using the bosonization formula, Eq. (93). But be careful: you will have to find first $\psi^{\dagger}(x+a)\left(-i \partial_{x}\right) \psi(x)$, normal-order it, expand to sub-leading order in $a$ (why?), subtract the vacuum expectation value and integrate, just like you did for : $\psi^{\dagger}(x) \psi(x)$ : before. Also, if you want to get the sub-leading term of or$\operatorname{der} \hat{N} / L$ in Eq. (106), you will have to work to sub-leading order in $1 / L$. This is a pretty long calculation.

\section{From the lattice to the linearized model}

We now want to make contact with the lattice model defined in the introduction. If we continue to forget about spin we have

$$
c_{j}=\sum_{k \in B Z} \frac{e^{i k j}}{\sqrt{L}} c_{k} .
$$

First note that the continuum limit ( $a \rightarrow 0$, where $a$ is the lattice spacing we set to 1 ) extends the Brillouin zone to $(-\infty \infty)$. We can then identify the continuous variable $x$ with $a j, x \rightarrow a j$, to have the physical field fermionic operator

$$
\psi_{\text {phys }}(x)=\sum_{k} \frac{e^{i k x}}{\sqrt{L}} c_{k} .
$$

By "physical" we mean that it relates to the long-wavelength part of the original fermions of, say, the Hubbard model. We can split this sum in two parts, corresponding to $k>0$ and $k<0$, and then shift each sum by $\pm k_{F}$, respectively, so that $k=0$ corresponds to the Fermi points

$$
\begin{aligned}
& \psi_{\text {phys }}(x)=\sum_{k>0} \frac{e^{i k x}}{\sqrt{L}} c_{k}+\sum_{k<0} \frac{e^{i k x}}{\sqrt{L}} c_{k}=\sum_{k=-k_{F}}^{\infty} \frac{e^{i\left(k+k_{F}\right) x}}{\sqrt{L}} c_{k+k_{F}}+\sum_{k=-\infty}^{k_{F}} \frac{e^{i\left(k-k_{F}\right) x}}{\sqrt{L}} c_{k-k_{F}} \\
& =e^{i k_{F} x}\left(\sum_{k=-k_{F}}^{\infty} \frac{e^{i k x}}{\sqrt{L}} c_{k+k_{F}}\right)+e^{-i k_{F} x}\left(\sum_{k=-\infty}^{k_{F}} \frac{e^{i k x}}{\sqrt{L}} c_{k-k_{F}}\right) \equiv e^{i k_{F} x} \psi_{R-p h y s}(x)+e^{-i k_{F} x} \psi_{L-p h y s}(x),(10
\end{aligned}
$$

where we introduced physical field operators for right- and left-movers.

We now make the jump of identifying the lattice model, at low energies, with the linearized dispersion model of Section III. The first thing to do is to let $k_{F} \rightarrow \infty$ in both terms above. We then have two branches of fermions, labeled 1 and 2, corresponding to each term in Eq. (109), and we make the identification

$$
\begin{aligned}
& 1 \longrightarrow R \\
& 2 \longrightarrow L .
\end{aligned}
$$

The first operation $(1 \rightarrow R)$ is quite straightforward. We define the species 1 of fermions by

$$
\begin{aligned}
c_{k}^{1} & =c_{k+k_{F}} \\
\psi^{1}(x) & =\sum_{k} \frac{e^{i k x}}{\sqrt{L}} c_{k}^{1}=\psi_{R}(x) \rightarrow \psi_{R-p h y s}(x),
\end{aligned}
$$

where, in the last equation, we dropped the "phys" label to show when we are working with the linear dispersion model. The second term above needs more care because, if we define $p=k+k_{F}$, then, for $p>0$ the states are filled, whereas for $p<0$, the states are empty in the ground state. This is reversed when compared with our previous definition. So we define instead

$$
\bar{k}=-\left(k+k_{F}\right) \Rightarrow\left\{\begin{array}{lll}
\bar{k} \leq 0 & \rightarrow & \text { filled } \\
\bar{k}>0 & \rightarrow & \text { empty }
\end{array}\right.
$$

Then

$$
\sum_{k} \frac{e^{i k x}}{\sqrt{L}} c_{k-k_{F}} \stackrel{k \rightarrow-k}{\longrightarrow} \sum_{k} \frac{e^{-i k x}}{\sqrt{L}} c_{-k-k_{F}} .
$$

We define a second species (2) of fermions such that

$$
\begin{aligned}
c_{k}^{2} & =c_{-k-k_{F}} \\
\psi^{2}(-x) & =\sum_{k} \frac{e^{-i k x}}{\sqrt{L}} c_{k}^{2}=\psi_{L}(x) \rightarrow \psi_{L-p h y s}(x) .
\end{aligned}
$$

Note the change of sign of $x$ for going from the 2-fermions to the left-moving fermions. The two kinds of fermions have the same dispersion

$$
\begin{aligned}
\varepsilon_{R}(k) & =v_{F}\left(k-k_{F}\right) \longrightarrow \varepsilon_{1}(k)=v_{F} k, \\
\varepsilon_{L}(k) & =-v_{F}\left(k+k_{F}\right) \longrightarrow \varepsilon_{2}(k)=v_{F} k,
\end{aligned}
$$

because of the definitions (110) and (112). We thus see that both 1 and 2 fermions are bona fide right-movers! We must attach the label $L$ or $R$, or 1 or 2 to each operator now. But, because of the sign change $x \rightarrow-x$ when $2 \rightarrow L$, we 
must make the same sign change in previous definitions if we want to work with $R$ and $L$, instead of 1 and 2 (which we do). So we list all the previous important formulas, for both
$R$ and $L$, with the appropriate changes and labels $\left(c_{k}^{2} \rightarrow c_{k}^{L}\right)$. Note that only the expressions that contain $x$ are modified, since in $k$-space, both species are right-movers.

$$
\begin{aligned}
& \psi_{R, L}(x)=\sum_{k} \frac{e^{ \pm i k x}}{\sqrt{L}} c_{k}^{R, L}, \\
& \left\{\psi_{R, L}(x), \psi_{R, L}(y)\right\}=\left\{\psi_{R, L}^{\dagger}(x), \psi_{R, L}^{\dagger}(y)\right\}=0, \\
& \left\{\psi_{R, L}(x), \psi_{R, L}^{\dagger}(y)\right\}=\sum_{n=-\infty}^{+\infty} \delta(x-y-n L) \\
& \rho_{R, L}(q)=\sum_{k} c_{k+q}^{R, L \dagger} c_{k}^{R, L}, \\
& \rho_{R, L}(q)= \begin{cases}\sqrt{\frac{L q}{2 \pi}} b_{q}^{R, L \dagger} & q>0, \\
\sqrt{\frac{L|q|}{2 \pi}} b_{-q}^{R, L} & q<0,\end{cases} \\
& : \psi_{R, L}^{\dagger}(x) \psi_{R, L}(x):=\frac{\hat{N}_{R, L}}{L}+\frac{1}{L} \sum_{q>0}\left[e^{\mp i q x} \rho_{R, L}(q)+e^{ \pm i q x} \rho_{R, L}(-q)\right] \text {, } \\
& =\frac{\hat{N}_{R, L}}{L}+\frac{1}{\sqrt{2 \pi L}} \sum_{q>0} \sqrt{q}\left[e^{ \pm i q x} b_{q}^{R, L}+e^{\mp i q x} b_{q}^{R, L \dagger}\right], \\
& \psi_{R, L}(x)=\frac{F_{R, L}}{\sqrt{L}} e^{ \pm i \frac{2 \pi \hat{N}_{R, L}}{L} x} \exp \left[\sum_{q>0} \alpha_{q}( \pm x) b_{q}^{R, L \dagger}\right] \exp \left[-\sum_{q>0} \alpha_{q}^{*}( \pm x) b_{q}^{R, L}\right] \\
& \varphi_{R, L}(x)=-\frac{i}{\sqrt{2 \pi}} \sum_{q>0} \alpha_{q}^{*}( \pm x) e^{-\alpha q / 2} b_{q}^{R, L}=\frac{i}{\sqrt{L}} \sum_{q>0} \frac{e^{ \pm i q x}}{\sqrt{q}} e^{-\alpha q / 2} b_{q}^{R, L}, \\
& \varphi_{R, L}^{\dagger}(x)=\frac{i}{\sqrt{2 \pi}} \sum_{q>0} \alpha_{q}( \pm x) e^{-\alpha q / 2} b_{q}^{R, L \dagger}=-\frac{i}{\sqrt{L}} \sum_{q>0} \frac{e^{\mp i q x}}{\sqrt{q}} e^{-\alpha q / 2} b_{q}^{R, L \dagger}, \\
& \phi_{R, L}(x)=\varphi_{R, L}(x)+\varphi_{R, L}^{\dagger}(x)=-\frac{i}{\sqrt{2 \pi}} \sum_{q>0} e^{-\alpha q / 2}\left[\alpha_{q}^{*}( \pm x) b_{q}^{R, L}-\alpha_{q}( \pm x) b_{q}^{R, L \dagger}\right] \\
& =\frac{i}{\sqrt{L}} \sum_{q>0} \frac{1}{\sqrt{q}} e^{-\alpha q / 2}\left[e^{ \pm i q x} b_{q}^{R, L}-e^{\mp i q x} b_{q}^{R, L \dagger}\right], \\
& \psi_{R, L}(x)=\frac{F_{R, L}}{\sqrt{L}} e^{ \pm i \frac{2 \pi \hat{N}_{R, L}}{L} x} \exp \left[-i \sqrt{2 \pi} \varphi_{R, L}^{\dagger}(x)\right] \exp \left[-i \sqrt{2 \pi} \varphi_{R, L}(x)\right], \\
& \psi_{R, L}(x)=\frac{F_{R, L}}{\sqrt{2 \pi \alpha}} e^{ \pm \frac{2 \pi \hat{N}_{R, L}}{L} x} \exp \left[-i \sqrt{2 \pi} \phi_{R, L}(x)\right] \\
& : \psi_{R, L}^{\dagger}(x) \psi_{R, L}(x):=\frac{\hat{N}_{R, L}}{L} \mp \frac{1}{\sqrt{2 \pi}} \partial_{x} \phi_{R, L} \text {, } \\
& H_{0}=v_{F} \sum_{q>0} \sum_{\nu=R, L} q b_{q}^{\nu \dagger} b_{q}^{\nu}+\frac{\pi}{L} v_{F} \hat{N}_{\nu}\left(\hat{N}_{\nu}+1\right), \\
& H_{0}=v_{F} \int_{-L / 2}^{L / 2} d x\left[: \psi_{R}^{\dagger}(x)\left(-i \partial_{x}\right) \psi_{R}(x):+: \psi_{L}^{\dagger}(x)\left(i \partial_{x}\right) \psi_{L}(x):\right] \text {, } \\
& H_{0}=\frac{v_{F}}{2} \sum_{\nu=R, L}\left[\int_{-L / 2}^{L / 2} d x:\left(\partial_{x} \phi_{\nu}\right)^{2}:+\frac{\pi}{L} \hat{N}_{\nu}\left(\hat{N}_{\nu}+1\right)\right] \text {. }
\end{aligned}
$$

The commutation relations of the bosonic field operators are (where $\approx$ means that we consider $|x-y| \ll L$ ) 


$$
\begin{aligned}
& {\left[\varphi_{R, L}(x), \varphi_{R, L}(y)\right] }=\left[\varphi_{R, L}^{\dagger}(x), \varphi_{R, L}^{\dagger}(y)\right]=0, \\
& {\left[\varphi_{R, L}(x), \varphi_{R, L}^{\dagger}(y)\right] }=-\frac{1}{2 \pi} \ln \left\{1-\exp \left[ \pm \frac{2 \pi i}{L}(x-y \pm i \alpha)\right]\right\} \\
& \approx-\frac{1}{2 \pi} \ln \left\{\mp \frac{2 \pi i}{L}(x-y \pm i \alpha)\right\} \mp \frac{i}{2 L}(x-y \pm i \alpha), \\
& {\left[\phi_{R, L}(x), \phi_{R, L}(y)\right] }=-\frac{1}{2 \pi} \ln \left\{\frac{1-\exp \left[ \pm \frac{2 \pi i}{L}(x-y \pm i \alpha)\right]}{1-\exp \left[\mp \frac{2 \pi i}{L}(x-y \mp i \alpha)\right]}\right\} \\
& \approx \pm \frac{i}{\pi} \arctan \left(\frac{x-y}{\alpha}\right) \mp \frac{i}{L}(x-y) \\
& \stackrel{\alpha \rightarrow 0}{\longrightarrow} \pm \frac{i}{2} \operatorname{sgn}(x-y) \mp \frac{i}{L}(x-y), \\
& {\left[\varphi_{R, L}(x), \partial_{y} \varphi_{R, L}^{\dagger}(y)\right] }= \pm \frac{i}{L} \frac{1}{1-\exp \left[\mp \frac{2 \pi i}{L}(x-y \pm i \alpha)\right]} \approx \pm \frac{1}{2 \pi} \frac{1}{x-y \pm i \alpha} \pm \frac{i}{2 L}, \\
& {\left[\partial_{x} \varphi_{R, L}(x), \varphi_{R, L}^{\dagger}(y)\right] }=\frac{i}{L} \frac{1}{1-\exp \left[\mp \frac{2 \pi i}{L}(x-y \pm i \alpha)\right]} \approx \frac{1}{2 \pi} \frac{1}{x-y \pm i \alpha} \mp \frac{i}{2 L}, \\
& {\left[\phi_{R, L}(x), \partial_{y} \phi_{R, L}(y)\right] }= \pm \frac{i}{L}\left\{\frac{1}{1-\exp \left[\mp \frac{2 \pi i}{L}(x-y \pm i \alpha)\right]}+\frac{1}{1-\exp \left[ \pm \frac{2 \pi i}{L}(x-y \pm i \alpha)\right]}\right\} \\
& \approx \mp \frac{i}{\pi} \frac{\alpha}{(x-y)^{2}+\alpha^{2}} \pm \frac{i}{L} \\
& \stackrel{\alpha \rightarrow 0}{\longrightarrow} \mp i \delta(x-y) \pm \frac{i}{L} .
\end{aligned}
$$

\section{Klein factors for more than one species}

There is one small modification we have to make in the developments of Sections III to X if we want to work with more than one species of fermions. That has to do with the Klein factors. The other operators, namely $b_{q}^{\nu}$ 's and $\hat{N}_{\nu}$ $(\nu=R, L)$ all commute between different branches so, for instance

$$
\begin{aligned}
{\left[b_{q}^{\nu}, b_{p}^{\nu^{\prime} \dagger}\right] } & =\delta_{p, q} \delta_{\nu, \nu^{\prime}} \\
{\left[\hat{N}_{\nu}, \hat{N}_{\nu^{\prime}}\right] } & =\delta_{\nu, \nu^{\prime}} .
\end{aligned}
$$

Remember how the Klein factors were defined. When acting on the $N$-particle ground state $|N\rangle_{0}$, we have

$$
\begin{aligned}
F|N\rangle_{0} & =|N-1\rangle_{0}, \\
F^{\dagger}|N\rangle_{0} & =|N+1\rangle_{0} .
\end{aligned}
$$

But, if there are two species of fermions, the corresponding $\left(N_{R}, N_{L}\right)$-particle ground states are tensor products (assuming $N_{R}$ and $N_{L}$ are both positive, the other case is analogous)

$$
\left|N_{R}, N_{L}\right\rangle_{0} \equiv\left|N_{R}\right\rangle_{0} \otimes\left|N_{L}\right\rangle_{0}=c_{N_{R}}^{R \dagger} c_{N_{R}-1}^{R \dagger} \cdots c_{1}^{R \dagger}|0\rangle_{0} \otimes c_{N_{L}}^{L \dagger} c_{N_{L}-1}^{L \dagger} \cdots c_{1}^{L \dagger}|0\rangle_{0} .
$$

Thus, to preserve the "fermionic" character of the annihilation/creation operators that makes them anti-commute between species, we have to define $F_{L}$ so that it picks up the sign coming from anti-commuting it past the right-moving fermionic operators

$$
\begin{aligned}
F_{L}\left|N_{R}, N_{L}\right\rangle_{0} & \equiv(-1)^{N_{R}}\left|N_{R}\right\rangle_{0} \otimes\left|N_{L}-1\right\rangle_{0}, \\
F_{L}^{\dagger}\left|N_{R}, N_{L}\right\rangle_{0} & \equiv(-1)^{N_{R}}\left|N_{R}\right\rangle_{0} \otimes\left|N_{L}+1\right\rangle_{0} .
\end{aligned}
$$

If there are more than two, say $M$, species, then one must define a particular order for the species $\left(N_{1}, N_{2}, \cdots, N_{M}\right)$ such that

$$
\left|N_{1}, N_{2}, \cdots, N_{M}\right\rangle_{0} \equiv\left|N_{1}\right\rangle_{0} \otimes\left|N_{2}\right\rangle_{0} \otimes \cdots\left|N_{M}\right\rangle_{0}
$$


and pick up the total sign change from the preceding fermionic operators

$$
\begin{aligned}
F_{\nu}\left|N_{1}, N_{2}, \cdots, N_{M}\right\rangle_{0} & \equiv(-1)^{\sum_{\mu=1}^{\nu-1} N_{\mu}}\left|N_{1}, N_{2}, \cdots, N_{\nu}-1, \cdots, N_{M}\right\rangle_{0} \\
F_{\nu}^{\dagger}\left|N_{1}, N_{2}, \cdots, N_{M}\right\rangle_{0} & \equiv(-1)^{\sum_{\mu=1}^{\nu-1} N_{\mu}}\left|N_{1}, N_{2}, \cdots, N_{\nu}+1, \cdots, N_{M}\right\rangle_{0}
\end{aligned}
$$

This situation is common when we add spin $(M=4)$. Naturally, the Klein factors still commute with $b_{q}^{\nu}$ 's. The main consequence of definitions (153) and (154) is that the Klein factors anti-commute with one another

$$
\begin{aligned}
{\left[b_{q}^{\nu}, F_{\nu^{\prime}}\right] } & =\left[b_{q}^{\nu \dagger}, F_{\nu^{\prime}}\right]=\left[b_{q}^{\nu}, F_{\nu^{\prime}}^{\dagger}\right]=\left[b_{q}^{\nu \dagger}, F_{\nu^{\prime}}^{\dagger}\right]=0 \\
\left\{F_{\nu}^{\dagger}, F_{\nu^{\prime}}\right\} & =2 \delta_{\nu, \nu^{\prime}} \\
\left\{F_{\nu}, F_{\nu^{\prime}}\right\} & =\left\{F_{\nu}^{\dagger}, F_{\nu^{\prime}}^{\dagger}\right\}=0,\left(\text { if } \nu \neq \nu^{\prime}\right)^{* * * *} \\
{\left[F_{\nu}, \hat{N}_{\nu^{\prime}}\right] } & =\delta_{\nu, \nu^{\prime}} F_{\nu}, \\
{\left[F_{\nu}^{\dagger}, \hat{N}_{\nu^{\prime}}\right] } & =-\delta_{\nu, \nu^{\prime}} F_{\nu}^{\dagger} .
\end{aligned}
$$

I draw attention to the starred equation above. Klein factors are not like fermionic creation/annihilation operators: their repeated action is not zero!

\section{The dual fields}

It is also very common in the field theory literature to define new so-called dual fields, even and odd combinations of $\phi_{R, L}$

$$
\begin{aligned}
\phi & =\frac{1}{\sqrt{2}}\left(\phi_{L}-\phi_{R}\right) \\
\theta & =\frac{1}{\sqrt{2}}\left(\phi_{L}+\phi_{R}\right)
\end{aligned} \Longrightarrow \begin{aligned}
\phi_{R} & =\frac{1}{\sqrt{2}}(\theta-\phi) \\
\phi_{L} & =\frac{1}{\sqrt{2}}(\theta+\phi)
\end{aligned}
$$

such that

$$
\begin{aligned}
\psi_{R, L}(x) & =\frac{F_{R, L}}{\sqrt{2 \pi \alpha}} e^{ \pm i \frac{2 \pi \hat{N}_{R, L}}{L} x} \exp [-i \sqrt{\pi}[\theta(x) \mp \phi(x)]] \\
: \psi_{R, L}^{\dagger}(x) \psi_{R, L}(x): & =\frac{\hat{N}_{R, L}}{L}+\frac{1}{2 \sqrt{\pi}} \partial_{x} \phi \mp \frac{1}{2 \sqrt{\pi}} \partial_{x} \theta \\
: \psi_{R}^{\dagger} \psi_{R}:+: \psi_{L}^{\dagger} \psi_{L}: & =\frac{\hat{N}_{R}+\hat{N}_{L}}{L}+\frac{1}{\sqrt{\pi}} \partial_{x} \phi \\
: \psi_{R}^{\dagger} \psi_{R}:-: \psi_{L}^{\dagger} \psi_{L}: & =-\frac{1}{\sqrt{\pi}} \partial_{x} \theta \\
{[\phi(x), \phi(y)] } & =[\theta(x), \theta(y)]=0, \\
{[\phi(x), \theta(y)] } & =-\frac{i}{2} \operatorname{sgn}(x-y)+\frac{i}{L}(x-y), \\
{\left[\phi(x), \partial_{y} \theta(y)\right] } & =i \delta(x-y)-\frac{i}{L} . \\
H_{0} & =\frac{v_{F}}{2} \int_{-L / 2}^{L / 2} d x\left[:\left(\partial_{x} \theta\right)^{2}:+:\left(\partial_{x} \phi\right)^{2}:\right]+\frac{\pi}{L} v_{F} \sum_{\nu=R, L} \hat{N}_{\nu}\left(\hat{N}_{\nu}+1\right)
\end{aligned}
$$

Note the similarity of the second to last equation (when $L \rightarrow \infty$ ) with a canonical commutation relation. Because of this, and because each of $\phi$ and $\theta$ commutes with itself at any $x$ and $y$, we define a canonical momentum field conjugate to $\phi(x)$

$$
\Pi(x)=\partial_{x} \theta(x) .
$$

The Hamiltonian can now be written in the canonical form

$$
H_{0}[\Pi(x), \phi(x)]=\frac{v_{F}}{2} \int_{-L / 2}^{L / 2} d x\left[:(\Pi)^{2}:+:\left(\partial_{x} \phi\right)^{2}:\right]+\frac{\pi}{L} v_{F} \sum_{\nu=R, L} \hat{N}_{\nu}\left(\hat{N}_{\nu}+1\right) .
$$


It is interesting to consider the Mattis-Mandelstam formula in terms of the dual fields (161). The exponential of $\theta(x)$ can be written

$$
e^{-i \sqrt{\pi} \int_{-L / 2}^{x} d y \Pi(y)}
$$

which, in light of the behavior of $\Pi(x)$ as a canonical momentum, can be viewed as a displacement operator for the $\phi$ field. In other words, it shifts the $\phi$-field configuration by a fixed amount of $\sqrt{\pi}$ from $-L / 2$ to $x$. This is a kink configuration. It is clear that the $x$ derivative of the kink is a delta function of strength $\sqrt{\pi}$ at $x$, which is consistent with the expression (163) of the fermionic density in terms of $\partial_{x} \phi$. Now, the other exponential in (161), $e^{ \pm i \sqrt{\pi} \phi(x)}$ is simply responsible for the transmutation of statistics. Without it, (171) is a bosonic operator and commutes with itself at different spatial points. The exponential $e^{ \pm i \sqrt{\pi} \phi(x)}$ ensures, through (167), that the combination anti-commutes at different locations, as can be easily checked.

\section{An interacting spinless model}

\section{XIV.1 The model and its solution}

We would now like to apply these ideas to a specific case. First recall that the fermionic operator is written as

$$
\begin{gathered}
\psi(x)=e^{i k_{F} x} \psi_{R-p h y s}(x)+e^{-i k_{F} x} \psi_{L-p h y s}(x) \\
\approx e^{i k_{F} x} \psi_{R}(x)+e^{-i k_{F} x} \psi_{L}(x) .
\end{gathered}
$$

A general local electron-electron interaction $\psi^{\dagger} \psi^{\dagger} \psi \psi$ will generate terms like $\psi_{R}^{\dagger} \psi_{R} \psi_{R}^{\dagger} \psi_{R}, \psi_{L}^{\dagger} \psi_{L} \psi_{L}^{\dagger} \psi_{L}$, $\psi_{R}^{\dagger} \psi_{R} \psi_{L}^{\dagger} \psi_{L}, e^{-2 i k_{F} x} \psi_{R}^{\dagger} \psi_{L} \psi_{R}^{\dagger} \psi_{R}$, and $e^{-4 i k_{F} x} \psi_{R}^{\dagger} \psi_{L} \psi_{R}^{\dagger} \psi_{L}$, and their Hermitian conjugates. Terms that contain oscillating exponentials, called Umklapp terms, average to zero and are usually neglected (unless $k_{F}$ commensurates with the underlying lattice and the exponential disappears; these cases will be dealt with later). We therefore focus on a "bare-bones" model that contains only the following interaction part

$$
H_{\text {int }}=\int_{-L / 2}^{L / 2} d x\left[\frac{g_{4}}{2} \sum_{\nu}:\left(: \psi_{\nu}^{\dagger} \psi_{\nu}:\right)^{2}:+g_{2}:\left(: \psi_{R}^{\dagger} \psi_{R}:: \psi_{L}^{\dagger} \psi_{L}:\right):\right]
$$

Note that we have to normal-order after multiplying two normal-ordered operators. This operation will not be usually indicated in what follows but is always implied. The interacting Hamiltonian (174) is known as the Luttinger model. Luttinger was the first to propose and solve it, albeit incorrectly [9]. Mattis and Lieb gave the correct solution shortly afterwards [10]. The nomenclature in terms of $g_{2}$ and $g_{4}$ is standard in the literature and is amusingly called "g-ology" $[5,4]$. The Hamiltonian (174) assumes a delta-function type of interaction. A more general longer range interaction can be used, but we will not do it here (see Ref. [15]). In terms of the bosonic field operators

$$
\begin{aligned}
H_{i n t} & =H_{i n t}^{a}+H_{i n t}^{b} \\
H_{i n t}^{a} & =\frac{1}{L}\left[\frac{g_{4}}{2}\left(\hat{N}_{R}^{2}+\hat{N}_{L}^{2}\right)+g_{2} \hat{N}_{R} \hat{N}_{L}\right] \\
H_{i n t}^{b} & =\int_{-L / 2}^{L / 2} \frac{d x}{2 \pi}\left[\frac{g_{4}}{2} \sum_{\nu}:\left(\partial_{x} \phi_{\nu}\right)^{2}:-g_{2}:\left(\partial_{x} \phi_{R}\right)\left(\partial_{x} \phi_{L}\right):\right]
\end{aligned}
$$

The terms linear in $\partial_{x} \phi_{\nu}$ integrate to zero. The non-interacting part is

$$
H_{0}=\frac{v_{F}}{2} \int_{-L / 2}^{L / 2} d x \sum_{\nu}:\left(\partial_{x} \phi_{\nu}\right)^{2}:+\frac{\pi v_{F}}{L} \sum_{\nu} \hat{N}_{\nu}^{2}
$$

We immediately see, in bosonic language, that the $g_{4}$-term only renormalizes the Fermi velocity

$$
\begin{aligned}
H=H_{0}+H_{\text {int }} & =\frac{v_{F}}{2}\left(1+\bar{g}_{4}\right) \int_{-L / 2}^{L / 2} d x\left[\sum_{\nu}:\left(\partial_{x} \phi_{\nu}\right)^{2}:-2 \frac{\bar{g}_{2}}{\left(1+\bar{g}_{4}\right)}:\left(\partial_{x} \phi_{R}\right)\left(\partial_{x} \phi_{L}\right):\right] \\
& +\frac{\pi v_{F}}{L}\left(1+\bar{g}_{4}\right)\left[\sum_{\nu} \hat{N}_{\nu}^{2}+2 \frac{\bar{g}_{2}}{\left(1+\bar{g}_{4}\right)} \hat{N}_{R} \hat{N}_{L}\right]
\end{aligned}
$$


where

$$
\begin{aligned}
\bar{g}_{2} & =\frac{g_{2}}{2 \pi v_{F}}, \\
\bar{g}_{4} & =\frac{g_{4}}{2 \pi v_{F}} .
\end{aligned}
$$

In $k$-space the Hamiltonian reads

$$
\begin{gathered}
H=H_{b}+H_{N} \\
H_{b}=v_{F}\left(1+\bar{g}_{4}\right) \sum_{q>0} q\left[\sum_{\nu} b_{q}^{\nu \dagger} b_{q}^{\nu}+\lambda\left(b_{q}^{R \dagger} b_{q}^{L \dagger}+\text { H.c. }\right)\right] \\
H_{N}=\frac{\pi v_{F}}{L}\left(1+\bar{g}_{4}\right)\left[\sum_{\nu} \hat{N}_{\nu}^{2}+2 \lambda \hat{N}_{R} \hat{N}_{L}\right],
\end{gathered}
$$

where $\lambda=\frac{\bar{g}_{2}}{\left(1+\bar{g}_{4}\right)}$. The astonishing feature of onedimensional systems is the fact that the interacting Hamiltonian (182) can be diagonalized exactly! This is achieved by the so-called Bogoliubov transformation. We will just state the results and leave the details for the Appendix D. In terms of new bosonic operators (we will use indices 1 and 2 instead of $R$ and $L$ for the new fields; not to be confused with the labels in Section XI)

$$
\begin{aligned}
d_{q}^{1} & =\cosh \gamma b_{q}^{R}+\sinh \gamma b_{q}^{L \dagger}, \\
d_{q}^{2 \dagger} & =\sinh \gamma b_{q}^{R}+\cosh \gamma b_{q}^{L \dagger}, \\
b_{q}^{R} & =\cosh \gamma d_{q}^{1}-\sinh \gamma d_{q}^{2 \dagger}, \\
b_{q}^{L \dagger} & =-\sinh \gamma d_{q}^{1}+\cosh \gamma d_{q}^{2 \dagger},
\end{aligned}
$$

such that

$$
\tanh 2 \gamma=\lambda \Rightarrow \gamma=\frac{1}{4} \ln \left(\frac{1+\lambda}{1-\lambda}\right)
$$

then Hamiltonian (182b) becomes

$$
H_{b}=v_{F}\left(1+\bar{g}_{4}\right) \sqrt{1-\lambda^{2}} \sum_{\nu=1,2} \sum_{q>0} q d_{q}^{\nu \dagger} d_{q}^{\nu}+\text { const. }
$$

The constant above is actually infinite but is subtracted out by normal ordering. It is convenient to define

$$
\begin{aligned}
g & \equiv \sqrt{\frac{1-\lambda}{1+\lambda}}=\sqrt{\frac{1+\bar{g}_{4}-\bar{g}_{2}}{1+\bar{g}_{4}+\bar{g}_{2}}} \\
u & \equiv v_{F}\left(1+\bar{g}_{4}\right) \sqrt{1-\lambda^{2}}=v_{F} \sqrt{\left(1+\bar{g}_{4}\right)^{2}-\bar{g}_{2}^{2}} .
\end{aligned}
$$

In the literature, one also finds $K=g$. It follows that

$$
H_{b}=u \sum_{\nu} \sum_{q>0} q d_{q}^{\nu \dagger} d_{q}^{\nu}
$$

and

$$
\begin{aligned}
& d_{q}^{1}=\frac{1}{2}\left[\left(\frac{1}{\sqrt{g}}+\sqrt{g}\right) b_{q}^{R}+\left(\frac{1}{\sqrt{g}}-\sqrt{g}\right) b_{q}^{L \dagger}\right] \\
& d_{q}^{2 \dagger}=\frac{1}{2}\left[\left(\frac{1}{\sqrt{g}}-\sqrt{g}\right) b_{q}^{R}+\left(\frac{1}{\sqrt{g}}+\sqrt{g}\right) b_{q}^{L \dagger}\right] .
\end{aligned}
$$

The inverse transformation is

$$
\begin{aligned}
& b_{q}^{R}=\frac{1}{2}\left[\left(\frac{1}{\sqrt{g}}+\sqrt{g}\right) d_{q}^{1}-\left(\frac{1}{\sqrt{g}}-\sqrt{g}\right) d_{q}^{2 \dagger}\right] \\
& b_{q}^{L \dagger}=\frac{1}{2}\left[-\left(\frac{1}{\sqrt{g}}-\sqrt{g}\right) d_{q}^{1}+\left(\frac{1}{\sqrt{g}}+\sqrt{g}\right) d_{q}^{2 \dagger}\right] .
\end{aligned}
$$

It is sometimes convenient to realize that the above transformation can be generated canonically with the unitary operator

$$
\begin{aligned}
U_{B} & =\exp \left[\gamma \sum_{q>0}\left(b_{q}^{R \dagger} b_{q}^{L \dagger}-b_{q}^{R} b_{q}^{L}\right)\right], \\
U_{B}^{-1} & =U_{B}^{\dagger}, \\
U_{B} b_{q}^{R} U_{B}^{-1} & =\frac{1}{2}\left[\left(\frac{1}{\sqrt{g}}+\sqrt{g}\right) b_{q}^{R}-\left(\frac{1}{\sqrt{g}}-\sqrt{g}\right) b_{q}^{L \dagger}\right], \\
U_{B} b_{q}^{L \dagger} U_{B}^{-1} & =\frac{1}{2}\left[-\left(\frac{1}{\sqrt{g}}-\sqrt{g}\right) b_{q}^{R}+\left(\frac{1}{\sqrt{g}}+\sqrt{g}\right) b_{q}^{L \dagger}\right] .
\end{aligned}
$$


After the canonical transformation, we must identify $b_{q}^{R, L} \rightarrow d_{q}^{1,2}$ to make contact with Eqs. (183-195). Besides, the fact that the transformation is canonical guarantees that the new operators $d_{q}^{\nu}$ obey bosonic commutation relations. It is convenient to define new bosonic field operators (though we still use $\varphi$ and $\phi$, the indices 1 and 2 will distinguish them from the ones related to $\left.b_{q}^{R, L}\right)$,

$$
\begin{aligned}
\varphi_{1,2}(x) & =\frac{i}{\sqrt{L}} \sum_{q>0} \frac{e^{ \pm i q x}}{\sqrt{q}} e^{-\alpha q / 2} d_{q}^{1,2}, \\
\varphi_{1,2}^{\dagger}(x) & =-\frac{i}{\sqrt{L}} \sum_{q>0} \frac{e^{\mp i q x}}{\sqrt{q}} e^{-\alpha q / 2} d_{q}^{1,2 \dagger}, \\
\phi_{1,2}(x) & =\varphi_{1,2}(x)+\varphi_{1,2}^{\dagger}(x) \\
& =\frac{i}{\sqrt{L}} \sum_{q>0} \frac{1}{\sqrt{q}} e^{-\alpha q / 2}\left[e^{ \pm i q x} d_{q}^{1,2}-e^{\mp i q x} d_{q}^{1,2 \dagger}\right] .
\end{aligned}
$$

For future use, take note of the following commutators, analogous to (134-136)

$$
\begin{aligned}
{\left[\varphi_{1,2}(x), \varphi_{1,2}(y)\right] } & =\left[\varphi_{1,2}^{\dagger}(x), \varphi_{1,2}^{\dagger}(y)\right]=0 \\
{\left[\varphi_{1,2}(x), \varphi_{1,2}^{\dagger}(y)\right] } & =-\frac{1}{2 \pi} \ln \left\{1-\exp \left[ \pm \frac{2 \pi i}{L}(x-y \pm i \alpha)\right]\right\} \\
& \approx-\frac{1}{2 \pi} \ln \left\{\mp \frac{2 \pi i}{L}(x-y \pm i \alpha)\right\} \mp \frac{i}{2 L}(x-y \pm i \alpha)
\end{aligned}
$$

From (183) and (184),

$$
\phi_{1,2}(x)=\cosh \gamma \phi_{R, L}-\sinh \gamma \phi_{L, R},
$$

and in terms of the canonical pair $\phi$ and $\theta$, by using (192) and (193)

$$
\begin{aligned}
\phi_{1,2} & =\frac{1}{\sqrt{2}}\left[\sqrt{g} \theta(x) \mp \frac{\phi(x)}{\sqrt{g}}\right], \\
\theta(x) & =\frac{1}{\sqrt{2 g}}\left[\phi_{1}(x)+\phi_{2}(x)\right], \\
\phi(x) & =\sqrt{\frac{g}{2}}\left[\phi_{2}(x)-\phi_{1}(x)\right] .
\end{aligned}
$$

In terms of these new fields, the diagonalized Hamiltonian is

$$
\begin{aligned}
H_{b} & =\frac{u}{2} \sum_{\nu=1,2} \int_{-L / 2}^{L / 2} d x:\left(\partial_{x} \phi_{\nu}\right)^{2}: \\
& =\frac{u}{2} \int_{-L / 2}^{L / 2} d x\left[g:\left(\partial_{x} \theta\right)^{2}:+\frac{1}{g}:\left(\partial_{x} \phi\right)^{2}:\right]
\end{aligned}
$$

Now, the reason for calling $\phi$ and $\theta$ as dual fields becomes transparent. The pairs $\left[\phi(x), \Pi_{\phi}(y)=\partial_{y} \theta(y)\right]$ and $\left[\theta(x), \Pi_{\theta}(y)=\partial_{y} \phi(y)\right]$ are both canonically conjugate and give equivalent, $\phi$ - or $\theta$-representations of (212), like the $x$ - and $p$-representations in quantum mechanics. If we define the coefficient of the squared derivative of the field in a given representation as the "coupling constant" $(1 / g$ in the $\phi$-representation, for example), then a weak coupling theory in one representation is "dual" (equivalent) to a strong coupling theory in the other and vice-versa. From (189), we see that $g<1$ corresponds to repulsive interactions and $g>1$ to attractive ones, $g=1$ being the non-interacting point. Thus, the above duality links repulsive and attractive cases and the non-interactive theory is self-dual.

Finally, the $H_{N}$ part is diagonalized by defining

$$
\begin{aligned}
\left\{\begin{array}{c}
\hat{N} \\
\hat{J}
\end{array}\right\} & =\hat{N}_{R} \pm \hat{N}_{L} \\
H_{N} & =\frac{\pi v_{F}}{2 L}\left(1+\bar{g}_{4}\right)\left[(1+\lambda) \hat{N}^{2}+(1-\lambda) \hat{J}^{2}\right] \\
& =\frac{\pi}{2 L} \frac{u}{\sqrt{1-\lambda^{2}}}\left[(1+\lambda) \hat{N}^{2}+(1-\lambda) \hat{J}^{2}\right] \\
& =\frac{\pi}{2 L}\left[\frac{u}{g} \hat{N}^{2}+u g \hat{J}^{2}\right]
\end{aligned}
$$

\section{XIV.2 Correlation functions of the spinless model}

We will now calculate some important correlation functions of Hamiltonian (179) at $T=0$. We start with the smooth part of the density (charge) 


$$
\begin{aligned}
& \hat{O}_{c}(x)=: \psi_{R}^{\dagger}(x) \psi_{R}(x):+: \psi_{L}^{\dagger}(x) \psi_{L}(x):=\frac{\hat{N}_{R}+\hat{N}_{L}}{L}+\frac{1}{\sqrt{2 \pi}}\left[\partial_{x} \phi_{L}(x)-\partial_{x} \phi_{R}(x)\right] \\
& =\frac{\hat{N}}{L}+\sqrt{\frac{g}{2 \pi}}\left[\partial_{x} \phi_{2}(x)-\partial_{x} \phi_{1}(x)\right]=\frac{\hat{N}}{L}+\sqrt{\frac{g}{2 \pi L}} \sum_{q>0} \sqrt{q} e^{-\alpha q / 2}\left[e^{-i q x}\left(d_{q}^{2}+d_{q}^{1 \dagger}\right)+e^{i q x}\left(d_{q}^{2 \dagger}+d_{q}^{1}\right)\right]
\end{aligned}
$$

where we used the inverse of Eq. (207). If we focus on the fluctuation part (second term), we write

$$
\begin{aligned}
D_{c}(x, y) & \equiv\left\langle\delta \hat{O}_{c}(x) \delta \hat{O}_{c}(y)\right\rangle \\
& =\frac{g}{2 \pi L} \sum_{p, q} \sqrt{p q} e^{-\alpha(p+q) / 2}\left[e^{-i(q x-p y)}\left\langle d_{q}^{2} d_{p}^{2 \dagger}+d_{q}^{1 \dagger} d_{p}^{1}\right\rangle+e^{i(q x-p y)}\left\langle d_{q}^{2 \dagger} d_{p}^{2}+d_{q}^{1} d_{p}^{1 \dagger}\right\rangle\right] \\
& =\frac{g}{2 \pi L} \sum_{q} q e^{-\alpha q}\left[e^{-i q(x-y)}+\text { c.c. }\right]=-\frac{g}{2 \pi^{2}(x-y)^{2}}
\end{aligned}
$$

We stress a few aspects of the above result. First, the expectation value is calculated with respect to the interacting ground state of Hamiltonian (191). Second, the crossed terms are obviously zero and only $q=p$ terms survive. Besides, we see that the power law decay of the correlation function occurs with exponent 2 , which is also the noninteracting value. Therefore, there is no renormalization by interactions of the power-law exponent of this correlation function. This will not occur in general, as we will see. Finally, the interactions only change the pre-factor of the power law. This actually gives the $q \rightarrow 0$ limit of the Fourier transform of the $D_{c}$ correlation function, which is related to the compressibility of the system.

We now focus on a different correlation function. The staggered part of the density is

$$
\begin{aligned}
\hat{O}_{C D W}(x) & \equiv e^{-2 i k_{F} x} \frac{e^{-i \frac{2 \pi}{L} \hat{N}_{R} x} F_{R}^{\dagger} F_{L} e^{-i \frac{2 \pi}{L} \hat{N}_{L} x}}{L} e^{i \sqrt{2 \pi} \varphi_{R}^{\dagger}(x)} e^{i \sqrt{2 \pi} \varphi_{R}(x)} e^{-i \sqrt{2 \pi} \varphi_{L}^{\dagger}(x)} e^{-i \sqrt{2 \pi} \varphi_{L}(x)} \\
& =e^{-2 i k_{F} x} e^{-i \frac{2 \pi}{L}\left(\hat{N}_{R}+\hat{N}_{L}+1\right) x} \frac{F_{R}^{\dagger} F_{L}}{L} e^{i \sqrt{2 \pi}\left[\varphi_{R}^{\dagger}(x)-\varphi_{L}^{\dagger}(x)\right]} e^{i \sqrt{2 \pi}\left[\varphi_{R}(x)-\varphi_{L}(x)\right]}
\end{aligned}
$$

which is now normal-ordered. We want to calculate the expectation value in the interacting ground state

$$
D_{C D W}(x-y)=\left\langle\hat{O}_{C D W}(x) \hat{O}_{C D W}^{\dagger}(y)+\text { H.c. }\right\rangle \text {. }
$$

The non-fluctuating part (Klein and number factors) is

$$
\begin{aligned}
& e^{-2 i k_{F}(x-y)}\left\langle e^{-i \frac{2 \pi}{L}\left(\hat{N}_{R}+\hat{N}_{L}+1\right) x} F_{R}^{\dagger} F_{L} F_{L}^{\dagger} F_{R} e^{i \frac{2 \pi}{L}\left(\hat{N}_{R}+\hat{N}_{L}+1\right) y}\right\rangle=e^{-2 i k_{F}(x-y)}\left\langle e^{-i \frac{2 \pi}{L}\left(\hat{N}_{R}+\hat{N}_{L}+1\right)(x-y)}\right\rangle \\
& =e^{-2 i k_{F}(x-y)}\left\langle e^{-i \frac{2 \pi}{L}(\hat{N}+1)(x-y)}\right\rangle \stackrel{L \rightarrow \infty}{\longrightarrow} e^{-2 i k_{F}(x-y)},
\end{aligned}
$$

where we assumed the number eigenvalue is not extensive. If we define $\bar{\varphi}(x) \equiv \sqrt{2 \pi}\left[\varphi_{R}(x)-\varphi_{L}(x)\right]$, we can simplify the notation to (we will suppress the oscillating exponential in intermediate steps)

$$
\hat{O}_{C D W}(x) \hat{O}_{C D W}^{\dagger}(y)=\frac{1}{L^{2}} e^{i \bar{\varphi}^{\dagger}(x)} e^{i \bar{\varphi}(x)} e^{-i \bar{\varphi}^{\dagger}(y)} e^{-i \bar{\varphi}(y)} .
$$

Using identity 2 of Appendix C, we can normal-order this expression

$$
\begin{gathered}
e^{i \bar{\varphi}(x)} e^{-i \bar{\varphi}^{\dagger}(y)}=e^{-i \bar{\varphi}^{\dagger}(y)} e^{i \bar{\varphi}(x)} e^{C}, \\
C=\left[\bar{\varphi}(x), \bar{\varphi}^{\dagger}(y)\right]=2 \ln \left\{\frac{L}{2 \pi \sqrt{(x-y)^{2}+\alpha^{2}}}\right\},
\end{gathered}
$$




$$
\Rightarrow \hat{O}_{C D W}(x) \hat{O}_{C D W}^{\dagger}(y)=\frac{1}{4 \pi^{2}(x-y)^{2}} e^{i\left[\bar{\varphi}^{\dagger}(x)-\bar{\varphi}^{\dagger}(y)\right]} e^{i[\bar{\varphi}(x)-\bar{\varphi}(y)]} .
$$

We now use the following strategy. By definition,

$$
\begin{gathered}
\bar{\varphi}(x)=i \sqrt{\frac{2 \pi}{L}} \sum_{q>0} \frac{1}{\sqrt{q}} e^{-\alpha q / 2}\left(e^{i q x} b_{q}^{R}-e^{-i q x} b_{q}^{L}\right), \\
\bar{\varphi}^{\dagger}(x)=i \sqrt{\frac{2 \pi}{L}} \sum_{q>0} \frac{1}{\sqrt{q}} e^{-\alpha q / 2}\left(e^{i q x} b_{q}^{L \dagger}-e^{-i q x} b_{q}^{R \dagger}\right) .
\end{gathered}
$$

In terms of the $d_{q}^{1,2}$ bosons we have

$$
\begin{aligned}
\bar{\varphi}(x) & =i \sqrt{\frac{2 \pi}{L}} \sum_{q>0} \frac{1}{\sqrt{q}} e^{-\alpha q / 2}\left[e^{i q x}\left(\cosh \gamma d_{q}^{1}-\sinh \gamma d_{q}^{2 \dagger}\right)-e^{-i q x}\left(-\sinh \gamma d_{q}^{1 \dagger}+\cosh \gamma d_{q}^{2}\right)\right] \\
& =\cosh \gamma \zeta(x)-\sinh \gamma \zeta^{\dagger}(x), \\
\bar{\varphi}^{\dagger}(x) & =\cosh \gamma \zeta^{\dagger}(x)-\sinh \gamma \zeta(x),
\end{aligned}
$$

where, by analogy with Eqs. (225), we defined

$$
\begin{gathered}
\zeta(x)=i \sqrt{\frac{2 \pi}{L}} \sum_{q>0} \frac{1}{\sqrt{q}} e^{-\alpha q / 2}\left(e^{i q x} d_{q}^{1}-e^{-i q x} d_{q}^{2}\right), \\
\zeta^{\dagger}(x)=i \sqrt{\frac{2 \pi}{L}} \sum_{q>0} \frac{1}{\sqrt{q}} e^{-\alpha q / 2}\left(e^{i q x} d_{q}^{2 \dagger}-e^{-i q x} d_{q}^{1 \dagger}\right) .
\end{gathered}
$$

We define now $V(x, y) \equiv \zeta(x)-\zeta(y)$ and find

$$
\begin{aligned}
\bar{\varphi}(x)-\bar{\varphi}(y) & =\cosh \gamma[\zeta(x)-\zeta(y)]-\sinh \gamma\left[\zeta^{\dagger}(x)-\zeta^{\dagger}(y)\right] \\
& =\cosh \gamma V(x, y)-\sinh \gamma V^{\dagger}(x, y)
\end{aligned}
$$

Inserting into Eq. (224),

$$
\begin{aligned}
\hat{O}_{C D W}(x) \hat{O}_{C D W}^{\dagger}(y) & =\frac{1}{4 \pi^{2}(x-y)^{2}} e^{i\left[\cosh \gamma V^{\dagger}-\sinh \gamma V\right]} e^{i\left[\cosh \gamma V-\sinh \gamma V^{\dagger}\right]} \\
& =\frac{1}{4 \pi^{2}(x-y)^{2}} e^{i\left[(\cosh \gamma-\sinh \gamma) V^{\dagger}+(\cosh \gamma-\sinh \gamma) V\right]} e^{-X / 2},
\end{aligned}
$$

where

$$
X=\left[\cosh \gamma V^{\dagger}-\sinh \gamma V, \cosh \gamma V-\sinh \gamma V^{\dagger}\right]=-\left[V, V^{\dagger}\right]
$$

We can normal order (230)

$$
: \hat{O}_{C D W}(x) \hat{O}_{C D W}^{\dagger}(y):=\frac{1}{4 \pi^{2}(x-y)^{2}} e^{i(\cosh \gamma-\sinh \gamma) V^{\dagger}} e^{i(\cosh \gamma-\sinh \gamma) V} e^{-Y / 2} e^{-X / 2},
$$

with

$$
Y=(\cosh \gamma-\sinh \gamma)^{2}\left[V, V^{\dagger}\right]
$$

Since $X$ and $Y$ are c-numbers

$$
X+Y=(\cosh 2 \gamma-\sinh 2 \gamma-1)\left[V, V^{\dagger}\right]=(g-1)\left[V, V^{\dagger}\right]
$$


Now

$$
\begin{aligned}
{\left[V, V^{\dagger}\right] } & =\left[\zeta(x)-\zeta(y), \zeta^{\dagger}(x)-\zeta^{\dagger}(y)\right] \\
& =\left[\zeta(x), \zeta^{\dagger}(x)\right]+\left[\zeta(y), \zeta^{\dagger}(y)\right]-\left[\zeta(x), \zeta^{\dagger}(y)\right]-\left[\zeta(y), \zeta^{\dagger}(x)\right] \\
& =4 \ln \left(\frac{L}{2 \pi \alpha}\right)-4 \ln \left(\frac{L}{2 \pi \sqrt{(x-y)^{2}+\alpha^{2}}}\right) \rightarrow 4 \ln \left|\frac{x-y}{\alpha}\right| .
\end{aligned}
$$

We made use of the fact that the commutator of $\zeta(x)$ is the same as the commutator of $\bar{\varphi}(x)$, Eq. (223). Finally, inserting into Eq. (234) and Eq. (232), we have

$$
\begin{aligned}
: \hat{O}_{C D W}(x) \hat{O}_{C D W}^{\dagger}(y): & =\frac{1}{4 \pi^{2}(x-y)^{2}}\left|\frac{\alpha}{x-y}\right|^{2(g-1)} e^{i(\cosh \gamma-\sinh \gamma) V^{\dagger}} e^{i(\cosh \gamma-\sinh \gamma) V} \\
& =\frac{1}{4 \pi^{2} \alpha^{2}}\left|\frac{\alpha}{x-y}\right|^{2 g} e^{i(\cosh \gamma-\sinh \gamma) V^{\dagger}} e^{i(\cosh \gamma-\sinh \gamma) V}
\end{aligned}
$$

Since the expression is now normal ordered, its vacuum expectation value is 1 . Putting back in the oscillating exponential and adding the Hermitian conjugate

$$
D_{C D W}(x) \propto \frac{\cos \left(2 k_{F} x\right)}{|x|^{2 g}}
$$

Here there is a non-trivial renormalization of the power law exponent by interactions. Half the exponent is called the anomalous dimension of the operator $\hat{O}_{C D W}$. It is anomalous because dimensional analysis would lead to the value of 2 , as can be seen from the prefactor $\alpha^{-2}$ of Eq. (236).

We leave as an exercise the calculation of the following correlation functions, which correspond to $4 k_{F}$ density oscillations and pairing (superconducting) correlations

$$
\begin{aligned}
\hat{O}_{4 k_{F}} \equiv e^{-4 i k_{F} x} \psi_{R}^{\dagger} \psi_{L} \psi_{R}^{\dagger} \psi_{L} & \Rightarrow\left\langle\hat{O}_{4 k_{F}}(x) \hat{O}_{4 k_{F}}^{\dagger}(x)+\text { H.c. }\right\rangle \propto \frac{\cos \left(4 k_{F} x\right)}{|x|^{8 g}} \\
\hat{O}_{p} \equiv \psi_{R}^{\dagger} \psi_{L} & \Rightarrow\left\langle\hat{O}_{p}(x) \hat{O}_{p}^{\dagger}(x)+\text { H.c. }\right\rangle \propto \frac{1}{|x|^{2 / g}} .
\end{aligned}
$$

It is actually more convenient to set up the Mattis-Mandelstam formula directly in terms of the "new" bosons. Thus, prove first that

$$
\begin{aligned}
\psi_{R, L}(x)= & \frac{F_{R, L}}{\sqrt{2 \pi \alpha}} e^{ \pm i \frac{2 \pi \hat{N}_{R, L}}{L} x}\left(\frac{2 \pi \alpha}{L}\right)^{\frac{1}{4}\left(\frac{1}{g}+g\right)} \exp \left\{-i \sqrt{2 \pi}\left[\cosh \gamma \varphi_{1,2}^{\dagger}(x)+\sinh \gamma \varphi_{2,1}^{\dagger}(x)\right]\right\} \times \\
& \exp \left\{-i \sqrt{2 \pi}\left[\cosh \gamma \varphi_{1,2}(x)+\sinh \gamma \varphi_{2,1}(x)\right]\right\}
\end{aligned}
$$

Also, calculate the single-particle Green's function

$$
i G\left(x, t_{x} ; y, t_{y}\right)=\left\langle T \psi\left(x, t_{x}\right) \psi^{\dagger}\left(y, t_{y}\right)\right\rangle_{0} \equiv\left\{\begin{array}{cl}
\left\langle\psi\left(x, t_{x}\right) \psi^{\dagger}\left(y, t_{y}\right)\right\rangle_{0} & \text { if } t_{x}>t_{y} \\
-\left\langle\psi^{\dagger}\left(y, t_{y}\right) \psi\left(x, t_{x}\right)\right\rangle_{0} & \text { if } t_{x}<t_{y} .
\end{array} .\right.
$$

The time dependence is introduced by means of the evolution operator $\hat{O}(t)=e^{i H t} \hat{O} e^{-i H t}$. The time dependence of the "new" bosons is obtained easily

$$
\begin{aligned}
& d_{q}^{1}(t)=e^{i u q t d_{q}^{1 \dagger} d_{q}^{1}} d_{q}^{1} e^{-i u q t d_{q}^{1 \dagger} d_{q}^{1}}=e^{-i u q t} d_{q}^{1}, \\
& d_{q}^{2}(t)=e^{-i u q t} d_{q}^{2} .
\end{aligned}
$$

It follows that the "new" bosonic field operators depend on time in a very special manner

$$
\begin{aligned}
& \varphi_{1,2}(x, t)=\frac{i}{\sqrt{L}} \sum_{q>0} \frac{e^{ \pm i q x}}{\sqrt{q}} e^{-\alpha q / 2} d_{q}^{1,2} e^{-i u q t} \\
& =\frac{i}{\sqrt{L}} \sum_{q>0} \frac{e^{ \pm i q(x \mp u t)}}{\sqrt{q}} e^{-\alpha q / 2} d_{q}^{1,2}=\varphi_{1,2}(x \mp u t) \equiv \varphi_{1,2}\left(x_{ \pm}\right) .
\end{aligned}
$$


We thus see that the complete time dependence of the bosonic operators can be introduced by making $x \rightarrow x+$ in the " 1 " boson and $x \rightarrow x_{-}$in the " 2 " boson. It is also quite clear why they are called chiral: they correspond to right- and left-moving waves, respectively. The Klein factors also acquire a time dependence, because the Hamiltonian contains number operators. They can be conveniently written together with the number phases as

$$
e^{i H t} F_{R, L} e^{ \pm \frac{2 \pi i}{L} \hat{N}_{R, L} x} e^{-i H t}=F_{R, L} \exp \left\{ \pm \frac{\pi i}{L}\left[\hat{N}\left(x \mp \frac{u}{g} t\right) \pm \hat{J}(x \mp u g t)\right]\right\} .
$$

Finally, by using the fact that

$$
\left\langle\psi\left(x, t_{x}\right) \psi^{\dagger}\left(y, t_{y}\right)\right\rangle_{0}=e^{i k_{F}(x-y)}\left\langle\psi_{R}\left(x, t_{x}\right) \psi_{R}^{\dagger}\left(y, t_{y}\right)\right\rangle_{0}+e^{-i k_{F}(x-y)}\left\langle\psi_{L}\left(x, t_{x}\right) \psi_{L}^{\dagger}\left(y, t_{y}\right)\right\rangle_{0},
$$

since the cross-term is zero, show that $G\left(x, t_{x} ; y, t_{y}\right)=\tilde{G}\left(x-y, t_{x}-t_{y}\right)$, where (neglecting the number eigenvalues in the thermodynamic limit)

$$
\tilde{G}(x, t)=\left(\frac{e^{i k_{F} x}}{2 \pi(x-u t)}-\frac{e^{-i k_{F} x}}{2 \pi(x+u t)}\right)\left(\frac{\alpha^{2}}{x^{2}-u^{2} t^{2}}\right)^{\frac{1}{4}\left(\frac{1}{g}+g-2\right)}
$$

We give a derivation of (247) for the more general case of electrons with spin in Appendix E. Two immediate and important consequences of Eq. (247) are the form of the local density of states and the momentum distribution of a onedimensional system

$$
\begin{aligned}
\rho_{\text {local }}(\omega) & \equiv \int_{-\infty}^{\infty} \frac{d t}{2 \pi} e^{i \omega t} \tilde{G}(x=0, t) \\
& \propto \int_{-\infty}^{\infty} \frac{d t}{2 \pi} e^{i \omega t} \frac{1}{t^{\nu}} \propto \omega^{\nu-1} \\
n_{R}(k) & \equiv \int_{-L / 2}^{L / 2} d x e^{-i k x} \tilde{G}_{R}\left(x, t=0^{-}\right) \\
& \sim \frac{1}{2}-C \operatorname{sgn}\left(k-k_{F}\right)\left|k-k_{F}\right|^{\nu-1}(24)
\end{aligned}
$$

where $C$ is a constant, $\nu=\frac{1}{2}\left(g+\frac{1}{g}\right) \geq 1$, and the equality holds at the non-interacting point $g=1$. We see that the local density of states is suppressed to zero as $\omega \rightarrow 0$, a result peculiar to one dimension. Also, the momentum distribution function does not have a jump at $k_{F}$ as it does in higher dimensions, but shows a power law non-analyticity at the Fermi wave-vector.

\section{The anisotropic Heisenberg (XXZ) model}

The XXZ spin- $\frac{1}{2}$ Hamiltonian has already appeared in Eq. (4) of Section II. We will set $J=1$ as the energy scale. There is a clever trick, due to Jordan and Wigner [24], that maps spin- $\frac{1}{2}$ operators to spinless fermions (it only works for $S=\frac{1}{2}$ ). We introduce fermionic operators $c_{j}$ and $c_{j}^{\dagger}$, with the usual anti-commutation relations

$$
\begin{aligned}
& \left\{c_{j}, c_{l}\right\}=\left\{c_{j}^{\dagger}, c_{l}^{\dagger}\right\}=0, \\
& \left\{c_{j}, c_{l}^{\dagger}\right\}=\delta_{j, l} .
\end{aligned}
$$

If we focus on one site only, then we can make the following mapping $\left(n_{j} \equiv c_{j}^{\dagger} c_{j}\right)$

$$
\begin{gathered}
S_{j}^{z} \rightarrow n_{j}-\frac{1}{2}, \\
S_{j}^{+} \equiv S_{j}^{x}+i S_{j}^{y} \rightarrow c_{j}^{\dagger}, \\
S_{j}^{-} \equiv S_{j}^{x}-i S_{j}^{y} \rightarrow c_{j},
\end{gathered}
$$

for it reproduces the usual spin- $\frac{1}{2}$ commutation relations

$$
\begin{gathered}
{\left[S_{j}^{+}, S_{j}^{z}\right]=-S_{j}^{+},} \\
{\left[S_{j}^{-}, S_{j}^{z}\right]=S_{j}^{-},} \\
{\left[S_{j}^{+}, S_{j}^{-}\right]=2 S_{j}^{z},}
\end{gathered}
$$

and $\sum_{a}\left(S_{j}^{a}\right)^{2}=\frac{3}{4}$, as can be readily checked. However, we run into trouble if we try to directly generalize (252) to different sites because, whereas spin operators belonging to different sites commute, fermionic operators anti-commute. We can cure that by attaching a string operator that changes the statistics! We write

$$
\begin{gathered}
S_{j}^{z} \rightarrow n_{j}-\frac{1}{2}, \\
S_{j}^{+} \rightarrow c_{j}^{\dagger} e^{i \pi \phi_{j}}, \\
S_{j}^{-} \rightarrow c_{j} e^{-i \pi \phi_{j}}, \\
\phi_{j} \equiv \sum_{l=1}^{j-1} n_{l} .
\end{gathered}
$$

Obviously, the string operators leave (253) unchanged, since they introduce number operators of different sites. For the same reason, Eqs. (253a) and (253b) are immediately generalized to different sites 


$$
\begin{aligned}
& {\left[S_{j}^{+}, S_{l}^{z}\right]=-\delta_{j, l} S_{j}^{+},} \\
& {\left[S_{j}^{-}, S_{l}^{z}\right]=\delta_{j, l} S_{j}^{-} .}
\end{aligned}
$$

The generalization of Eq. (253c) depends crucially on the string operators

$$
\left[S_{j}^{+}, S_{l}^{-}\right]=c_{j}^{\dagger} e^{i \pi \phi_{j}} e^{-i \pi \phi_{l}} c_{l}-c_{l} e^{-i \pi \phi_{l}} e^{i \pi \phi_{j}} c_{j}^{\dagger}= \pm\left(c_{j}^{\dagger} c_{l}+c_{l} c_{j}^{\dagger}\right) e^{i \pi \phi_{j}} e^{-i \pi \phi_{l}}=0 \quad(j \neq l),
$$

where the upper (lower) sign occurs if $l>j(l<j)$. The string operators are able to introduce the appropriate sign change that turns a commutator into an anti-commutator, thus correcting for the different statistics.

We can now use the mapping (254) in the Hamiltonian (4). The XY part is

$$
\begin{aligned}
& S_{j}^{x} S_{j+1}^{x}+S_{j}^{y} S_{j+1}^{y}=\frac{1}{2}\left(S_{j}^{+} S_{j+1}^{-}+S_{j}^{-} S_{j+1}^{+}\right)=\frac{1}{2}\left(c_{j}^{\dagger} e^{i \pi \phi_{j}} e^{-i \pi \phi_{j+1}} c_{j+1}+c_{j} e^{-i \pi \phi_{j}} e^{i \pi \phi_{j+1}} c_{j+1}^{\dagger}\right) \\
& =\frac{1}{2}\left(c_{j}^{\dagger} e^{-i \pi n_{j}} c_{j+1}+c_{j} e^{i \pi n_{j}} c_{j+1}^{\dagger}\right)=\frac{1}{2}\left(c_{j}^{\dagger} c_{j+1}-c_{j} c_{j+1}^{\dagger}\right)=\frac{1}{2}\left(c_{j}^{\dagger} c_{j+1}+\text { H.c. }\right),
\end{aligned}
$$

where we used $c_{j} e^{i \pi n_{j}}=-c_{j}$ and $c_{j}^{\dagger} e^{i \pi n_{j}}=c_{j}^{\dagger}$. This is like a hopping term. Thus,

$$
H_{X X Z}=\frac{1}{2} \sum_{j}\left(c_{j}^{\dagger} c_{j+1}+\text { H.c. }\right)+\Delta \sum_{j}\left(n_{j}-\frac{1}{2}\right)\left(n_{j+1}-\frac{1}{2}\right) .
$$

If we use periodic boundary conditions, we must identify $\mathbf{S}_{L+1}$ with $\mathbf{S}_{1}$. The end term will be

$$
\begin{aligned}
& S_{L}^{x} S_{L+1}^{x}+S_{L}^{y} S_{L+1}^{y}=\frac{1}{2}\left(S_{L}^{+} S_{1}^{-}+S_{L}^{-} S_{1}^{+}\right)=\frac{1}{2}\left(c_{L}^{\dagger} e^{i \pi \phi_{L}} c_{1}+c_{L} e^{-i \pi \phi_{L}} c_{1}^{\dagger}\right) \\
& =\frac{\exp \left(i \pi \sum_{l=2}^{L-1} n_{l}\right)}{2}\left(c_{L}^{\dagger} c_{1}-c_{L} c_{1}^{\dagger}\right)=\frac{e^{i \pi(N+1)}}{2}\left(c_{L}^{\dagger} c_{1}+c_{1}^{\dagger} c_{L}\right),
\end{aligned}
$$

where $N$ is the total number of fermions. A phase is therefore left at the chain end. It is also convenient to transform the hopping term by doing a gauge transformation

$$
\begin{aligned}
c_{j} & \rightarrow e^{i \pi j} c_{j}, \\
c_{k} & \rightarrow \sum_{j=1}^{L} e^{-i(k-\pi) j} c_{j}=c_{k-\pi},
\end{aligned}
$$

such that

$$
\begin{aligned}
c_{j}^{\dagger} c_{j+1} & \rightarrow c_{j}^{\dagger} e^{-i \pi j} e^{i \pi(j+1)} c_{j+1}=-c_{j}^{\dagger} c_{j+1} \\
c_{L}^{\dagger} c_{1} & \rightarrow c_{L}^{\dagger} e^{-i \pi L} e^{i \pi} c_{1}=e^{i \pi(1-L)} c_{L}^{\dagger} c_{1} .
\end{aligned}
$$

The effect of the transformation is to change the sign of the hopping term while shifting the momenta by $\pi$. The chain end hopping has a phase $e^{i \pi(N-L)}$. Consequently, the physics of the model is invariant if we change the sign of the hopping term, which corresponds to $J \rightarrow-J, \Delta \rightarrow$ $-\Delta$ in the original model (up to a momentum shift of $\pi$ ). Physically, this corresponds to a rotation of the spins by $\pi$ around the z-axis at every other site. The above choice of a negative sign is convenient because then the dispersion is $\varepsilon(k)=-\cos k$, like we had in Section II of these lectures.
Note that the total z-axis magnetization is

$$
M=\sum_{j=1}^{L} S_{j}^{z}=N-\frac{L}{2} .
$$

We will mostly be working in sector $M=0$, where $N=\frac{L}{2}$. The end phase is then $e^{i \pi L / 2}$ and we choose $L$ even, so that the phase disappears. The Fermi wave vector is

$$
k_{F}=\pi n=\frac{\pi}{2},
$$

which corresponds to a half-filled band. The Fermi velocity will be $v_{F}=\sin k_{F}=1$.

The first thing we notice is that the model at $\Delta=0$ is trivially solved by the Jordan-Wigner transformation, since we end up with non-interacting fermions. This is the socalled XY model. We will not dwell further on it. When $\Delta \neq 0$, we get an interacting fermionic system.

We are now in a position to apply the bosonization technique to attack the model at $\Delta \neq 0$. We first linearize around $\pm k_{F}$ to get two branches of fermions which we let run from $-\infty$ to $\infty$. The fermionic field operator is

$$
\psi(x) \approx e^{i k_{F} x} \psi_{R}(x)+e^{-i k_{F} x} \psi_{L}(x) .
$$


The XY part is by now familiar

$$
H_{X Y} \approx \sum_{k, \nu} k: c_{k \nu}^{\dagger} c_{k \nu}:=\int_{-L / 2}^{L / 2} d x\left[: \psi_{R}^{\dagger}(x)\left(-i \partial_{x}\right) \psi_{R}(x):+: \psi_{L}^{\dagger}(x)\left(i \partial_{x}\right) \psi_{L}(x):\right] .
$$

In order to bosonize the interaction, we need an expression for $S_{j}^{z}=c_{j}^{\dagger} c_{j}-\frac{1}{2}$. First note that in the zero magnetization sector $\left\langle n_{j}\right\rangle_{0}=1 / 2$, so that

$$
S_{j}^{z} \approx: \psi^{\dagger}(x) \psi(x): \text {. }
$$

Using Eq. (267),

$$
S_{j}^{z} \approx \sum_{\nu}: \psi_{\nu}^{\dagger}(x) \psi_{\nu}(x):+e^{-2 i k_{F} x} \psi_{R}^{\dagger}(x) \psi_{L}(x)+\text { H.c. }
$$

The crucial thing to notice is that $S_{j}^{z}$ has a smooth and a rapidly oscillating part. The latter oscillates like

$$
e^{-2 i k_{F} x}=e^{-i \pi x}=(-1)^{j} .
$$

The interaction term is $\left(\rho_{\nu}(x) \equiv: \psi_{\nu}^{\dagger}(x) \psi_{\nu}(x):\right)$

$$
\begin{aligned}
S_{j}^{z} S_{j+1}^{z} & \approx\left[\rho_{R}+\rho_{L}+(-1)^{j} \psi_{R}^{\dagger} \psi_{L}+\text { H.c. }\right]\left[\rho_{R}+\rho_{L}+(-1)^{j+1} \psi_{R}^{\dagger} \psi_{L}+\text { H.c. }\right] \\
& =\left(\rho_{R}+\rho_{L}\right)^{2}-\psi_{R}^{\dagger} \psi_{L} \psi_{L}^{\dagger} \psi_{R}-\psi_{L}^{\dagger} \psi_{R} \psi_{R}^{\dagger} \psi_{L}-\left(\psi_{R}^{\dagger} \psi_{L}\right)^{2}+\text { H.c. } \\
& =\rho_{R}^{2}+\rho_{L}^{2}+4 \rho_{R} \rho_{L}-\left(\psi_{R}^{\dagger} \psi_{L}\right)^{2}+\text { H.c. }
\end{aligned}
$$

The first two terms correspond to the kind of interaction we dealt with before. The last term is an Umklapp term and we will ignore it for now. Thus, we get an effective model which is just the Luttinger model. The g-ology is

$$
\begin{aligned}
& g_{2}=4 \Delta, \\
& g_{4}=2 \Delta .
\end{aligned}
$$

Remembering the whole procedure is only valid when $\Delta \ll 1$, we get

$$
\begin{aligned}
& g=\sqrt{\frac{1+\bar{g}_{4}-\bar{g}_{2}}{1+\bar{g}_{4}+\bar{g}_{2}}}=\sqrt{\frac{1+\frac{\Delta}{\pi}-\frac{2 \Delta}{\pi}}{1+\frac{\Delta}{\pi}+\frac{2 \Delta}{\pi}}} \approx 1-\frac{2 \Delta}{\pi}, \\
& u=\sqrt{\left(1+\bar{g}_{4}\right)^{2}-\bar{g}_{2}^{2}} \approx 1+\frac{\Delta}{\pi} .
\end{aligned}
$$

In order to calculate correlation functions we also need an expression for $S_{j}^{ \pm}$, which means we have to bosonize the string operator

$$
\phi_{j}=\sum_{l=1}^{j-1} n_{l} \approx \int_{-L / 2}^{x} d y\left[\sum_{\nu}: \psi_{\nu}^{\dagger}(y) \psi_{\nu}(y):+\frac{1}{2}\right],
$$

where we have neglected the rapidly oscillating term in the integration. Using the expression of the density in terms of bosonic field operators, we obtain

$$
\phi_{j} \rightarrow \frac{x}{2}+\frac{1}{\sqrt{\pi}} \phi(x)+\text { const. }=\frac{\mathrm{x}}{2}+\frac{1}{\sqrt{2 \pi}}\left[\phi_{\mathrm{L}}(\mathrm{x})-\phi_{\mathrm{R}}(\mathrm{x})\right]+\text { const }
$$

which is conveniently local. We will neglect the constant as it is a boundary effect that goes away in the thermodynamic limit. By symmetrizing the string operator to make it hermitian and remembering $k_{F}=\frac{\pi}{2}$

$$
\begin{aligned}
S_{j}^{-} & \approx\left[e^{i k_{F} x} \psi_{R}(x)+e^{-i k_{F} x} \psi_{L}(x)\right]\left[e^{-i k_{F} x} e^{-i \sqrt{\frac{\pi}{2}}\left(\phi_{L}-\phi_{R}\right)}+\text { H.c. }\right] \\
& \approx\left[e^{i k_{F} x} F_{R} e^{-i \sqrt{2 \pi} \phi_{R}}+e^{-i k_{F} x} F_{L} e^{-i \sqrt{2 \pi} \phi_{L}}\right]\left[e^{-i k_{F} x} e^{-i \sqrt{\frac{\pi}{2}}\left(\phi_{L}-\phi_{R}\right)}+\text { H.c. }\right] \\
& \approx F_{R}\left[e^{-i \sqrt{\frac{\pi}{2}}\left(\phi_{L}+\phi_{R}\right)}+e^{2 i k_{F} x} e^{-i \sqrt{\frac{\pi}{2}}\left(3 \phi_{R}-\phi_{L}\right)}\right]+F_{L}\left[e^{-i \sqrt{\frac{\pi}{2}}\left(\phi_{R}+\phi_{L}\right)}+e^{-2 i k_{F} x} e^{-i \sqrt{\frac{\pi}{2}}\left(3 \phi_{L}-\phi_{R}\right)}\right] \\
& \approx e^{-i \sqrt{\pi} \theta}\left\{F_{R}\left[1+(-1)^{j} e^{i 2 \sqrt{\pi} \phi}\right]+F_{L}\left[1+(-1)^{j} e^{-i 2 \sqrt{\pi} \phi}\right]\right\}
\end{aligned}
$$


where we have been cavalier about normalization factors which do not affect the long distance physics.

We will simply state the results of and leave as an exercise the calculation of the following correlation functions [12]

$$
\begin{aligned}
G_{z z}(x, t) & =\left\langle S^{z}(x, t) S^{z}(0,0)\right\rangle_{0}, \\
G_{+-}(x, t) & =\left\langle S^{+}(x, t) S^{-}(0,0)\right\rangle_{0}
\end{aligned}
$$

Each one consists of a smooth and a rapidly oscillating part. Let us start by $G_{z z}$

$$
\begin{aligned}
G_{z z}(x, t)= & \frac{1}{\pi}\left\langle\partial_{x} \phi(x, t) \partial_{x} \phi(0,0)\right\rangle_{0}+(-1)^{j}\left\langle\psi_{R}^{\dagger}(x, t) \psi_{L}(x, t) \psi_{L}^{\dagger}(0,0) \psi_{R}(0,0)\right\rangle_{0} \\
& +(-1)^{j}\left\langle\psi_{L}^{\dagger}(x, t) \psi_{R}(x, t) \psi_{R}^{\dagger}(0,0) \psi_{L}(0,0)\right\rangle_{0} .
\end{aligned}
$$

We have already calculated the static limit of the first term. Its dynamic generalization is straightforward and gives

$$
G_{z z}^{1}(x, t)=-\frac{g}{4 \pi^{2}}\left[\frac{1}{(x-u t)^{2}}+\frac{1}{(x+u t)^{2}}\right]
$$

The second term was also calculated in the static limit when we found $D_{C D W}(x)$. The dynamical result is

$$
G_{z z}^{2}(x, t) \propto \frac{(-1)^{j}}{\left|x^{2}-u^{2} t^{2}\right|^{g}}
$$

Collecting everything

$$
G_{z z}(x, t)=-\frac{g}{4 \pi^{2}}\left[\frac{1}{(x-u t)^{2}}+\frac{1}{(x+u t)^{2}}\right]+\text { const. } \frac{(-1)^{\mathrm{j}}}{\left|\mathrm{x}^{2}-\mathrm{u}^{2} \mathrm{t}^{2}\right|^{\mathrm{g}}} .
$$

The transverse part also has two contributions due to the smooth and oscillating parts of $S^{ \pm}$(Eq. (279)) given by

$$
G_{+-}(x, t)=\frac{\text { const. }}{\left|x^{2}-u^{2} t^{2}\right|^{1 / 4 g}}+\frac{(-1)^{j} \text { const. }}{\left|x^{2}-u^{2} t^{2}\right|^{(-1+g+1 / 4 g)}}\left[\frac{1}{(x-u t)^{2}}+\frac{1}{(x+u t)^{2}}\right] .
$$

We note in passing that restoring the initial sign of the $\mathrm{XY}$ Hamiltonian results in multiplying Eq. (279) by $(-1)^{j}$ and the consequent transfer of the same factor from the second to the first term of Eq. (286).

\section{Haldane's Luttinger liquid con- jecture}

Everything we have said is valid perturbatively $\Delta \ll 1$. Haldane has conjectured $[15,16]$, based on renormalization group arguments, that the Luttinger model Hamiltonian we analyzed in the Section XIV is valid even away from the perturbative region, although the values of the interaction constants $g_{2}$ and $g_{4}$ have a different expression in terms of $\Delta$. In fact, his conjecture should be valid for just about any one-dimensional interacting gapless system. This is almost universally believed to be true and has been checked for several models. In the case of the XXZ model in Eq. (4), the system is gapless for $-1<\Delta \leq 1$ and the conjecture is believed to hold in this interval. Actually, it is known from the exact solution that [12]

$$
g=\frac{\pi}{2(\pi-\arccos \Delta)} .
$$

It reduces to the perturbative result (275) when $\Delta \ll 1$. Haldane's conjecture is actually a bit more predictive since the low energy effective model involves three parameters, namely the velocities $u, v_{N}=\frac{u}{g}$ and $v_{J}=u g$,

$$
H_{e f f}=u \sum_{\nu} \sum_{q>0} q d_{q}^{\nu \dagger} d_{q}^{\nu}+\frac{\pi}{2 L}\left[v_{N} \hat{N}^{2}+v_{J} \hat{J}^{2}\right]
$$

but the bosonization produced only two parameters $u$ and $g$. Thus, the three velocities bear a fixed relation

$$
u^{2}=v_{N} v_{J}
$$

Conversely, the correlation exponent $g$ can be obtained if the velocities are known

$$
g^{2}=\frac{v_{J}}{v_{N}} .
$$

One-dimensional gapless systems whose low-energy properties can be described by the Hamiltonian (288) such that (289) holds are called Luttinger liquids. This is because they bear a relation with the Luttinger model that is analogous to 
the one between the free Fermi gas and Landau's Fermi liquid theory $[25,26,27]$ of a higher dimensional $(D>1)$ interacting fermionic system.

It is interesting to note that the isotropic point $\Delta=1$ corresponds to $g=1 / 2$, where the two correlation functions (285) and (286) coincide (after transferring the $(-1)^{j}$ from the second to the first term of (286)), as they should.

\section{An interacting model of spin- $\frac{1}{2}$ fermions}

\section{XVII.1 The model and its solution}

We now want to generalize what we have been doing to electrons with spin $S=\frac{1}{2}$. With the knowledge that we have accumulated the task is quite straightforward. We introduce the following interacting Hamiltonian

$$
\begin{aligned}
H_{i n t}= & \int_{-L / 2}^{L / 2} d x\left\{\frac{1}{2} \sum_{\nu \sigma \sigma^{\prime}}\left[\left(g_{4 \|} \delta_{\sigma \sigma^{\prime}}+g_{4 \perp} \delta_{\sigma,-\sigma^{\prime}}\right):\left(: \psi_{\nu \sigma}^{\dagger} \psi_{\nu \sigma}:\right)\left(: \psi_{\nu \sigma^{\prime}}^{\dagger} \psi_{\nu \sigma^{\prime}}:\right):\right]\right. \\
& \left.+\sum_{\sigma \sigma^{\prime}}\left(g_{2 \|} \delta_{\sigma \sigma^{\prime}}+g_{2 \perp} \delta_{\sigma,-\sigma^{\prime}}\right):\left(: \psi_{R \sigma}^{\dagger} \psi_{R \sigma}:: \psi_{L \sigma^{\prime}}^{\dagger} \psi_{L \sigma^{\prime}}:\right):\right\}
\end{aligned}
$$

where $\sigma, \sigma^{\prime}= \pm$ now label spin projections along an arbitrary $z$-axis and we have for generality introduced different coupling constants for parallel $(\|)$ and anti-parallel $(\perp)$ spin interactions. The SU(2) symmetric model has $g_{i \|}=g_{i \perp}$. This is a Luttinger model for spin- $\frac{1}{2}$ electrons. It can be solved by bosonization. One simply introduces bosons $b_{q \sigma}^{R, L}$, number operators $\hat{N}_{\sigma}^{R, L}$, and Klein factors $F_{R, L \sigma}$ with additional spin indices, but with completely analogous definitions and properties (see Sections III to XIII). Obviously, operators with different spin indices commute, except for Klein factors, which anti-commute (Section XIII).

First, if we write

$$
\rho_{\nu \sigma} \equiv: \psi_{\nu \sigma}^{\dagger} \psi_{\nu \sigma}:
$$

then we can define charge and spin combinations

$$
\begin{aligned}
& \rho_{\nu}^{c} \equiv \frac{1}{\sqrt{2}}\left(\rho_{\nu+}+\rho_{\nu-}\right), \\
& \rho_{\nu}^{s} \equiv \frac{1}{\sqrt{2}}\left(\rho_{\nu+}-\rho_{\nu-}\right),
\end{aligned}
$$

such that

$$
\rho_{\nu \sigma}=\frac{1}{\sqrt{2}}\left(\rho_{\nu}^{c}+\sigma \rho_{\nu}^{s}\right) .
$$

It is easy to show that

$$
\begin{aligned}
\sum_{\sigma} \rho_{\nu \sigma} \rho_{\nu^{\prime} \sigma} & =\rho_{\nu}^{c} \rho_{\nu^{\prime}}^{c}+\rho_{\nu}^{s} \rho_{\nu^{\prime}}^{s}, \\
\sum_{\sigma} \rho_{\nu \sigma} \rho_{\nu^{\prime}-\sigma} & =\rho_{\nu}^{c} \rho_{\nu^{\prime}}^{c}-\rho_{\nu}^{s} \rho_{\nu^{\prime}}^{s}
\end{aligned}
$$

Inserting into the interacting Hamiltonian (291) results in

$$
\begin{aligned}
H_{\text {int }}= & \int_{-L / 2}^{L / 2} d x\left\{\frac{1}{2} \sum_{\nu}\left[\left(g_{4 \|}+g_{4 \perp}\right) \rho_{\nu}^{c} \rho_{\nu}^{c}+\left(g_{4 \|}-g_{4 \perp}\right) \rho_{\nu}^{s} \rho_{\nu}^{s}\right]\right. \\
& \left.+\left[\left(g_{2 \|}+g_{2 \perp}\right) \rho_{R}^{c} \rho_{L}^{c}+\left(g_{2 \|}-g_{2 \perp}\right) \rho_{R}^{s} \rho_{L}^{s}\right]\right\} .
\end{aligned}
$$

We can define the charge and spin combinations of the coupling constants

$$
\begin{aligned}
g_{i c} & \equiv\left(g_{i \|}+g_{i \perp}\right) \\
g_{i s} & \equiv\left(g_{i \|}-g_{i \perp}\right)
\end{aligned}
$$

In the presence of SU(2) symmetry, $g_{i s}=0$. The interacting
Hamiltonian now reads

$$
H_{\text {int }}=\int_{-L / 2}^{L / 2} d x \sum_{\lambda=c, s}\left[\frac{g_{4 \lambda}}{2} \sum_{\nu} \rho_{\nu}^{\lambda} \rho_{\nu}^{\lambda}+g_{2 \lambda} \rho_{R}^{\lambda} \rho_{L}^{\lambda}\right] \text {. }
$$

Obviously, definitions (293) and (294) induce analogous combinations of number and bosonic operators, both in $q$ 
and $x$ space

$$
\begin{aligned}
\hat{N}_{\nu(c, s)} & \equiv \frac{1}{\sqrt{2}}\left(\hat{N}_{\nu+} \pm \hat{N}_{\nu-}\right) \\
b_{q(c, s)}^{\nu} & \equiv \frac{1}{\sqrt{2}}\left(b_{q+}^{\nu} \pm b_{q-}^{\nu}\right)
\end{aligned}
$$

etc., such that operators with different $\lambda$ labels commute.

$$
\begin{aligned}
H_{i n t} & =H_{i n t}^{a}+H_{i n t}^{b}, \\
H_{i n t}^{a} & =\sum_{\lambda}\left\{\frac{1}{L}\left[\frac{g_{4 \lambda}}{2}\left(\hat{N}_{R \lambda}^{2}+\hat{N}_{L \lambda}^{2}\right)+g_{2 \lambda} \hat{N}_{R \lambda} \hat{N}_{L \lambda}\right]\right\}, \\
H_{i n t}^{b} & =\sum_{\lambda} \int_{-L / 2}^{L / 2} \frac{d x}{2 \pi}\left[\frac{g_{4 \lambda}}{2} \sum_{\nu}:\left(\partial_{x} \phi_{\nu \lambda}\right)^{2}:-g_{2 \lambda}:\left(\partial_{x} \phi_{R \lambda}\right)\left(\partial_{x} \phi_{L \lambda}\right):\right] .
\end{aligned}
$$

The non-interacting part can also be written in terms of charge and spin sectors as can be easily checked

$$
H_{0}=\sum_{\lambda}\left\{\frac{v_{F}}{2} \int_{-L / 2}^{L / 2} d x \sum_{\nu}:\left(\partial_{x} \phi_{\nu \lambda}\right)^{2}:+\frac{\pi v_{F}}{L} \sum_{\nu} \hat{N}_{\nu \lambda}^{2}\right\} .
$$

We now have two decoupled sectors corresponding to charge and spin excitations whose solution in terms of rotated bosons is analogous to what has already been done before for spinless fermions.

Let us briefly list a few of the important aspects of the full solution. There will now be a velocity $u_{\lambda}$ and a coupling constant $g_{\lambda}$ (or alternatively $K_{\lambda}$ ) for each sector $\lambda=c, s$ given by

$$
\begin{aligned}
g_{\lambda} & \equiv \sqrt{\frac{2 \pi v_{F}+g_{4 \lambda}-g_{2 \lambda}}{2 \pi v_{F}+g_{4 \lambda}+g_{2 \lambda}}} \\
u_{\lambda} & \equiv v_{F} \sqrt{\left(1+\frac{g_{4 \lambda}}{2 \pi v_{F}}\right)^{2}-\left(\frac{g_{2 \lambda}}{2 \pi v_{F}}\right)^{2}}
\end{aligned}
$$

Note that the SU(2) symmetric case is characterized by $g_{s}=1$ and $u_{s}=v_{F}$. The diagonalized Hamiltonian is given

$$
H=\sum_{\nu \lambda} u_{\lambda} \sum_{q>0} q d_{q \lambda}^{\nu \dagger} d_{q \lambda}^{\nu}+\frac{\pi}{2 L}\left[v_{N \lambda} \hat{N}_{\lambda}^{2}+v_{J \lambda} \hat{J}_{\lambda}^{2}\right]
$$

where the new bosons are defined in an obvious manner and

$$
\begin{aligned}
N_{\lambda} & \equiv N_{R \lambda}+N_{L \lambda}, \\
J_{\lambda} & \equiv N_{R \lambda}-N_{L \lambda}, \\
v_{N \lambda} & \equiv \frac{u_{\lambda}}{g_{\lambda}}, \\
v_{J \lambda} & \equiv u_{\lambda} g_{\lambda} .
\end{aligned}
$$

Note the same fixed relation between velocities

$$
u_{\lambda}^{2}=v_{N \lambda} v_{J \lambda}
$$

That the dynamics of the Luttinger model can be written in terms of decoupled charge and spin sectors, together with Haldane's conjecture that the universality class of gapless systems in one dimension is given at low energies by the Luttinger model dynamics, leads to the phenomenon of spin-charge separation, an important feature of fermionic systems in one spatial dimension. An electron that is introduced into an interacting system will rapidly decay into its constituent elementary excitations: charge and spin density modes that propagate at different velocities $\left(u_{c}\right.$ and $u_{s}$ ) and that will spatially separate with time. This phenomenon is completely absent in higher dimensions, at least in so far as they are described by Landau's Fermi liquid theory.

We note in passing that the Hubbard model, discussed at the beginning of these lectures has

$$
g_{2 \|}=g_{2 \perp}=g_{4 \|}=g_{4 \perp}=U
$$

at weak coupling $U \ll t$, such that

$$
\begin{aligned}
g_{c} & \approx 1-\frac{U}{\pi v_{F}} \\
u_{c} & \approx v_{F}\left(1+\frac{U}{\pi v_{F}}\right) \\
g_{s} & =1 \\
u_{s} & =v_{F}<u_{c} .
\end{aligned}
$$

At strong and intermediate couplings, the full dependences of both $g_{c}, u_{c}$, and $u_{s}$ on $U$ and the electron density $n$ can be obtained from the exact solution [28]. Although $g_{s}=1$ exactly, due to spin rotation invariance, $u_{s}$ is not given by $v_{F}$. The reason for this lies in the renormalization group flow from high to low energies and is outside the scope of these lectures. 


\section{XVII.2 Correlation functions of the spin-1/2 model}

The stage is now set for the calculation of any correlation functions we would like. We are aided by the fact that the spin and charge dynamics decouple and so do the fac- tors that involve only spin and charge fields. As this is fairly analogous to the spinless case and poses no new conceptual difficulty we will skip the calculational details. We will only give the normal ordered form of the Mattis-Mandelstam formula in terms of the new bosons

$$
\begin{aligned}
\psi_{R, L \sigma}(x)= & \frac{F_{R, L \sigma}}{\sqrt{2 \pi \alpha}} e^{ \pm \frac{2 \pi i}{L} \hat{N}_{R, L \sigma} x}\left(\frac{2 \pi \alpha}{L}\right)^{\frac{1}{8}\left(g_{c}+g_{s}+\frac{1}{g_{c}}+\frac{1}{g_{s}}\right)} \times \\
& \exp -i \sqrt{\pi}\left\{c_{c} \varphi_{1,2 c}^{\dagger}(x)+s_{c} \varphi_{2,1 c}^{\dagger}(x)+\sigma\left[c_{s} \varphi_{1,2 s}^{\dagger}(x)+s_{s} \varphi_{2,1 s}^{\dagger}(x)\right]\right\} \times \\
& \exp -i \sqrt{\pi}\left\{c_{c} \varphi_{1,2 c}(x)+s_{c} \varphi_{2,1 c}(x)+\sigma\left[c_{s} \varphi_{1,2 s}(x)+s_{s} \varphi_{2,1 s}(x)\right]\right\}
\end{aligned}
$$

where we used the short notation

$$
\begin{aligned}
& c_{\lambda} \equiv \cosh \gamma_{\lambda}, \\
& s_{\lambda} \equiv \sinh \gamma_{\lambda} .
\end{aligned}
$$

The definition of the new bosonic operators $\varphi_{1,2 \lambda}(x)$ is induced by (293) and (294) and is quite obvious.

We can then define operators analogous to the spinless case

$$
\begin{aligned}
\hat{O}_{c}(x) & =\sum_{\nu \sigma}: \psi_{\nu \sigma}^{\dagger}(x) \psi_{\nu \sigma}(x): \\
\hat{O}_{C D W}(x) & =\sum_{\sigma} e^{-2 i k_{F} x} \psi_{R \sigma}^{\dagger}(x) \psi_{L \sigma}(x) \\
\hat{O}_{4 k_{F}}(x) & =\sum_{\sigma} e^{-4 i k_{F} x} \psi_{R \sigma}^{\dagger}(x) \psi_{R-\sigma}^{\dagger}(x) \psi_{L-\sigma}(x) \psi_{L \sigma}(x),
\end{aligned}
$$

and others unique to the spin- $\frac{1}{2}$ case, describing the smooth and oscillating parts of the spin density

$$
\begin{aligned}
\hat{O}_{s}(x) & =\sum_{\nu \rho \tau} \sigma_{\rho \tau}^{3}: \psi_{\nu \rho}^{\dagger}(x) \psi_{\nu \tau}(x): \\
\hat{O}_{S D W}^{a}(x) & =\sum_{\rho \tau} e^{-2 i k_{F} x} \sigma_{\rho \tau}^{a} \psi_{R \rho}^{\dagger}(x) \psi_{L \tau}(x),
\end{aligned}
$$

where $\sigma_{\rho \tau}^{a}$ is the $\rho, \tau$ element of the $a$-th Pauli matrix $(\rho, \tau= \pm 1, a=1,2,3)$. Finally, pairing operators can be defined with specific spin symmetries,

$$
\begin{aligned}
\hat{O}_{p}^{S}(x) & =i \sum_{\rho \tau} \sigma_{\rho \tau}^{2} \psi_{R \rho}(x) \psi_{L \tau}(x) \\
\hat{O}_{p}^{T 0}(x) & =i \sum_{\rho \tau}\left(\sigma^{2} \sigma^{3}\right)_{\rho \tau} \psi_{R \rho}(x) \psi_{L \tau}(x) \\
\hat{O}_{p}^{T \pm 1}(x) & =i \sum_{\rho \tau}\left[\sigma^{2}\left(\sigma^{1} \pm i \sigma^{2}\right)\right]_{\rho \tau} \psi_{R \rho}(x) \psi_{L \tau}(x)
\end{aligned}
$$

Eq. (329) corresponds to singlet superconductivity (like the usual BCS pairing) while Eqs. (330) and (331) describe triplet superconducting correlations with total pair spin projection $S_{z}=0$ and $S_{z}= \pm 1$, respectively. The correlation functions are defined in the usual way

$$
D_{j}(x) \equiv\left\langle\hat{O}_{j}(x) \hat{O}_{j}^{\dagger}(0)\right\rangle_{0}
$$


They are

$$
\begin{aligned}
D_{c}(x) & =\frac{g_{c}}{(\pi x)^{2}}, \\
D_{C D W}(x) & \sim \frac{\cos \left(2 k_{F} x\right)}{x^{g_{c}+g_{s}}}, \\
D_{4 k_{F}}(x) & \sim \frac{\cos \left(4 k_{F} x\right)}{x^{4 g_{c}}}, \\
D_{s}(x) & =\frac{g_{s}}{(\pi x)^{2}}, \\
D_{S D W}^{z}(x) & \sim \frac{\cos \left(2 k_{F} x\right)}{x^{g_{c}+g_{s}}}, \\
D_{S D W}^{x, y}(x) & \sim \frac{\cos \left(2 k_{F} x\right)}{x^{\left(g_{c}+\frac{1}{g_{s}}\right)}}, \\
D_{p}^{S}(x) & =\frac{D_{p}^{T 0}(x) \sim}{x^{\left(\frac{1}{g_{c}}+g_{s}\right)}}, \\
D_{p}^{T \pm 1}(x) & \sim \frac{1}{x^{\left(\frac{1}{g_{c}}+\frac{1}{g_{s}}\right)}} .
\end{aligned}
$$

The $q \rightarrow 0$ limits of the Fourier transforms of Eqs. (333) and (336) give the bulk charge and spin susceptibilities of the system, the former being related to the compressibility.
When the system is $\mathrm{SU}(2)$ symmetric and $g_{s}=1$, all three components of $D_{S D W}^{a}(x)$ are equal and so are the singlet and triplet pairing correlation functions, as it should be.

The above correlation functions give information about the tendency of the system to show long range order. The latter is forbidden in one dimension when the operator in question has a continuous symmetry such as happens for the spin (SU(2)) and the pairing (U(1)) correlations. Nevertheless, the correlations that decay slowest are the dominant ones. Remembering that $g_{c}<1$ for repulsive interactions and $g_{c}>1$ for attractive ones and focusing on the SU(2) symmetric case, we see that attraction favors pairing (superconducting) correlations, whereas repulsion favors CDW or SDW correlations. Still in the SU(2) symmetric case, $4 k_{F}$ correlations dominate over CDW ones when $g_{c}<\frac{1}{3}$. The degeneracy between singlet and triplet pairing when there is attraction and between SDW and CDW when there is repulsion is lifted by sub-leading logarithmic corrections to these correlation functions, which we will not discuss [29, 30].

Finally, it is interesting to look at the single particle Green's function, defined in Section XIV. It is diagonal in spin indices and is given by

$\tilde{G}_{\sigma}(x, t)=\frac{\operatorname{sgn}(t)}{2 \pi}\left[\frac{e^{i k_{F} x}}{\sqrt{\left(x-u_{c} t\right)\left(x-u_{s} t\right)}}-\frac{e^{-i k_{F} x}}{\sqrt{\left(x+u_{c} t\right)\left(x+u_{s} t\right)}}\right]\left(\frac{\alpha^{2}}{x^{2}-u_{c}^{2} t^{2}}\right)^{\frac{1}{8}\left(g_{c}+\frac{1}{g_{c}}-2\right)}\left(\frac{\alpha^{2}}{x^{2}-u_{s}^{2} t^{2}}\right)^{\frac{1}{8}\left(g_{s}+\frac{1}{g_{s}}-2\right)}$.

This is derived in Appendix E. Note, once again that the anomalous dimension is $\frac{1}{8}\left(g_{\lambda}+\frac{1}{g_{\lambda}}-2\right)$. As a consequence, there will be is the usual anomalous power laws in the momentum distribution and local density of states, characteristic of one-dimensional systems

$$
\begin{aligned}
& \tilde{G}(x, t=0) \sim \frac{1}{x^{\nu}} \quad \Rightarrow \quad n_{R}(k)=\frac{1}{2}-C \operatorname{sgn}\left(k-k_{F}\right)\left|k-k_{F}\right|^{\nu-1}, \\
& \tilde{G}(x=0, t) \sim \frac{1}{t^{\nu}} \quad \Rightarrow \quad \rho_{\text {local }}(\omega) \sim \omega^{\nu-1}
\end{aligned}
$$

where

$$
\nu=\frac{1}{4} \sum_{\lambda}\left(g_{\lambda}+\frac{1}{g_{\lambda}}\right)
$$

\section{Gaps and the sine-Gordon the- ory}

We will now only mention an important aspect of the bosonized theories we have treated so far. Let us start with the XXZ model. In the bosonization of the Hamiltonian, we ignored the Umklapp terms

$$
H_{U m k l a p p}=\int d x\left[\left(\psi_{R}^{\dagger} \psi_{L}\right)^{2}+\text { H.c. }\right] \text {. }
$$

To leading order (forgetting Klein and number factors), the bosonization version is given by

$$
\begin{aligned}
H_{\text {Umklapp }} & =\frac{\Delta}{4 \pi^{2} \alpha^{2}} \int d x\left[e^{-i 4 \sqrt{\pi} \phi(x)}+\text { H.c. }\right] \\
& \sim \frac{\Delta}{2 \pi^{2} \alpha^{2}} \int d x \cos [4 \sqrt{\pi} \phi(x)] .
\end{aligned}
$$

The second form is rather cavalier with respect to Klein factors but is frequently used and contains the important part of the physics. Similar terms occur in the Hubbard model. For instance, we could write

$$
H_{b a c k}=g_{1 \perp} \sum_{\sigma} \int d x: \psi_{R \sigma}^{\dagger} \psi_{L \sigma} \psi_{L-\sigma}^{\dagger} \psi_{R-\sigma}:
$$




$$
H_{U m k l a p p}=g_{3 \perp} \sum_{\sigma} \int d x e^{-4 i k_{F} x}: \psi_{R \sigma}^{\dagger} \psi_{R-\sigma}^{\dagger} \psi_{L-\sigma} \psi_{L \sigma}+\text { H. c. : . }
$$

(Here, we finally see where the other indices 1 and 3 have gone!) Eq. (348) corresponds to a scattering event across the Fermi surface with an accompanying spin flip and is called a spin backscattering or simply backscattering term. Eq. (349), an Umklapp term involving different spins, has an oscillating factor, but at half-filling in the Hubbard model, for example, this factor disappears (the factor of two comes from the spin)

$$
k_{F}=\frac{\pi n}{2}=\frac{\pi}{2} \rightarrow e^{-4 i k_{F} x}=1 .
$$

When bosonized Eqs. (348) and (349) become

$$
\begin{aligned}
H_{\text {back }} & \sim g_{1 \perp} \int d x \cos \left[2 \sqrt{2 \pi} \phi_{s}(x)\right], \\
H_{\text {Umklapp }} & \sim g_{3 \perp} \int d x \cos \left[2 \sqrt{2 \pi} \phi_{c}(x)\right],
\end{aligned}
$$

which are quite similar to (347).

Let us look at the effect of such cosine terms. We focus on Eq. (347). We saw in Section XIV that the theory in the absence of this term is described by the Hamiltonians (211) or (212), where $\phi$ and $\partial_{x} \theta$ are canonically conjugate variables

$$
\left[\phi(x), \partial_{y} \theta(y)\right]=i \delta(x-y) .
$$

We can make the following canonical transformation

$$
\begin{aligned}
\phi & \rightarrow \sqrt{g} \phi, \\
\theta & \rightarrow \frac{\theta}{\sqrt{g}},
\end{aligned}
$$

which leaves (353) unchanged. In this case, the Umklapp term becomes

$$
H_{U m k l a p p} \rightarrow \frac{\Delta}{2 \pi^{2} \alpha^{2}} \int d x \cos [4 \sqrt{\pi g} \phi(x)] .
$$

It is known from renormalization group arguments that for $g>g_{\text {crit }}=1 / 2$, the Umklapp term is irrelevant: its effect on the low-energy sector is simply to renormalize the effective parameters $u$ and $g$ but, other than this, it can be ignored. However, if $g<1 / 2$, then the Umklapp term is relevant: it is responsible for the opening of a gap in the spectrum. In this case, a Luttinger liquid description is no longer valid. The case $g=g_{\text {crit }}=1 / 2$ (which coincides with the isotropic Heisenberg model as we saw) is a marginal case. The most important effect of (356) is the generation of logarithmic corrections to the power laws of the spin correlation functions [29, 30]. Thus, for the Heisenberg model

$$
\left\langle\mathbf{S}_{0} \cdot \mathbf{S}_{j}\right\rangle \propto(-1)^{j} \frac{\sqrt{\ln |j|}}{|j|}
$$

From the exact expression of $g$ in terms of $\Delta$ of the XXZ model given in Eq. (287) of Section XV, we can see that $g \in(1 / 2, \infty)$ if $-1<\Delta<1$, and the system is a Luttinger liquid. For $\Delta>1, g<1 / 2$ and (356) opens a gap in the spectrum. All this is corroborated by the exact solution.

This discussion can be easily generalized to the spinful case. Indeed, the terms in Eqs. (351) and (352) are relevant and generate a gap in the spin or charge spectra if $g_{s}<1$ or $g_{c}<1$, respectively. Let us discuss the physics of each of these cases separately. In a model with on-site interactions only, the Umklapp term occurs only at half-filling because of the commensurability condition (350). Then, for repulsive interactions $\left(g_{c}<1\right)$, there is a gap in the charge sector. Indeed, this is observed in the Hubbard model, in which any finite $U$ opens a gap at half-filling [20]. The spin sector remains gapless and is a Luttinger liquid.

On the other hand, spin backscattering (Eq. (351)) is relevant in the negative $U$ Hubbard model, which describes attractive on-site interactions. This is a spin-rotationally invariant model, so $g_{i \|}=g_{i \perp}$. Thus, there is a $g_{1 \|}$ term in addition to (351). It is fairly easy to show that this kind of term can be incorporated into a $g_{2}$-type interaction by $g_{2 \|} \rightarrow g_{2 \|}-g_{1 \|}$, such that both $g_{2 c} \rightarrow g_{2 c}-g_{1 \|}$, and $g_{2 s} \rightarrow g_{2 s}-g_{1 \|}$, effectively generating a $g_{s}<1$. This is what is needed for (351) to be relevant and open a spin gap. Indeed, the negative-U Hubbard model is known to possess a spin gap at any filling. The charge sector remains gapless with $g_{c}>1$, with dominant superconducting (singlet) correlations. This is the closest to a superconductor we can get in a one-dimensional system, since true long-range superconducting order is rigorously forbidden in $1 \mathrm{D}$. There is a particular case of the sine-Gordon model, the value $g_{s}=1 / 2$, where one can refermionize the system (i.e., map it back to a different fermionic system) and solve it exactly [31]! Because this solution was found by Luther and Emery, this spin-gapped quasi-superconducting phase is commonly referred to as the Luther-Emery phase.

\section{A Useful formulas}

\section{A.1 Sums in the first Brillouin zone}

$$
\begin{aligned}
& \sum_{k \in B Z} e^{i k j}=\sum_{n=0}^{L-1} e^{i \frac{2 \pi}{L} n j} \\
& =\sum_{n=0}^{L-1} x^{n}= \begin{cases}L & \text { if } x=1 \\
\frac{1-x^{L}}{1-x} & \text { if } \mathrm{x} \neq 1 .\end{cases}
\end{aligned}
$$


where $x=e^{i \frac{2 \pi j}{L}}$. It follows that

$$
\sum_{k \in B Z} e^{i k j}=L \delta_{j, 0} \cdot
$$

Analogously

$$
\begin{aligned}
\sum_{j=1}^{L} e^{i k j} & =\sum_{j=1}^{L} e^{i \frac{2 \pi}{L} n j} \\
& = \begin{cases}L & \text { if } x=1 \\
\frac{1-x^{L}}{1-x} & \text { if } \mathrm{x} \neq 1 .\end{cases}
\end{aligned}
$$

It follows that

$$
\sum_{j=1}^{L} e^{i k j}=L \delta_{k, 0}
$$

\section{A.2 Periodic delta-function}

Consider the following "saw-tooth" function, defined on the real axis

$$
f(x)=n-\frac{1}{2}-x \text { if } x \in(n-1, n),
$$

where $n \in \mathbb{Z}$ (see Fig. 6).

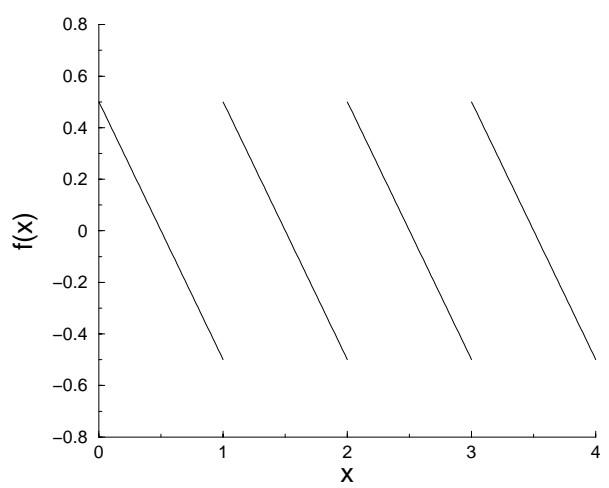

Figure 6. "Saw-tooth" function, defined in Eq. (360).

Its Fourier series can be written

$$
f(x)=\sum_{k=-\infty}^{\infty} e^{i 2 \pi k x} f_{k},
$$

where

$$
\begin{gathered}
f_{k}=\int_{0}^{1} e^{-i 2 \pi k x} f(x) d x \\
=\int_{0}^{1} e^{-i 2 \pi k x}\left(\frac{1}{2}-x\right) d x=\left\{\begin{array}{cc}
0 & \text { if } k=0, \\
\frac{1}{2 \pi i k} & \text { if } k \neq 0 .
\end{array}\right.
\end{gathered}
$$

So

$$
f(x)=\frac{1}{2 \pi i} \sum_{\substack{k=-\infty \\ k \neq 0}}^{\infty} \frac{e^{i 2 \pi k x}}{k} .
$$

Taking its derivative

$$
\frac{\partial f}{\partial x}=\sum_{k=-\infty}^{\infty} e^{i 2 \pi k x}-1 .
$$

But from its definition

$$
\frac{\partial f}{\partial x}=-1+\sum_{n=-\infty}^{\infty} \delta(x-n) .
$$

Thus,

$$
\sum_{k=-\infty}^{\infty} e^{i 2 \pi k x}=\sum_{n=-\infty}^{\infty} \delta(x-n)
$$

\section{B Bosonic coherent states}

Suppose there is only one boson $b\left(\left[b, b^{\dagger}\right]=1\right)$. Then, the state $e^{\alpha b^{\dagger}}|0\rangle$ is an eigenstate of $b$, since from identity 1 of Appendix C

$$
\begin{aligned}
e^{-\alpha b^{\dagger}} b e^{\alpha b^{\dagger}} & =b+\alpha \\
\Rightarrow b e^{\alpha b^{\dagger}} & =e^{\alpha b^{\dagger}}(b+\alpha) \\
\Rightarrow b e^{\alpha b^{\dagger}}|0\rangle & =\alpha e^{\alpha b^{\dagger}}|0\rangle .
\end{aligned}
$$

Note that $e^{\alpha b^{\dagger}}|0\rangle$ is not normalized. Actually, using identity 2 of Appendix C

$$
e^{\alpha^{*} b} e^{\alpha b^{\dagger}}=e^{\alpha b^{\dagger}} e^{\alpha^{*} b} e^{|\alpha|^{2}},
$$

and

$$
\left\langle 0\left|e^{\alpha^{*} b} e^{\alpha b^{\dagger}}\right| 0\right\rangle=e^{|\alpha|^{2}}\left\langle 0\left|e^{\alpha b^{\dagger}} e^{\alpha^{*} b}\right| 0\right\rangle=e^{|\alpha|^{2}} .
$$

Thus

$$
|\alpha\rangle=e^{-\frac{|\alpha|^{2}}{2}} e^{\alpha b^{\dagger}}|0\rangle
$$

is normalized. For different bosons labeled by $q$ the coherent states are

$$
\left|\left\{\alpha_{q}\right\}\right\rangle=\exp \left[\sum_{q}\left(-\frac{\left|\alpha_{q}\right|^{2}}{2}+\alpha_{q} b_{q}^{\dagger}\right)\right]|0\rangle .
$$

\section{Useful operators identities}

1. Baker-Hausdorff formula

$$
\begin{gathered}
e^{-B} A e^{B}=\sum_{n=0}^{\infty} \frac{1}{n !}[A, B]_{n} \\
=A+[A, B]+\frac{1}{2 !}[[A, B], B]+\cdots,
\end{gathered}
$$

where $[A, B]_{n}=\left[[A, B]_{n-1}, B\right]$ and $[A, B]_{0}=A$.

2. If $C \equiv[A, B]$ and $[A, C]=[B, C]=0$, then $e^{A} e^{B}=e^{A+B} e^{C / 2}$ and $e^{A} e^{B}=e^{B} e^{A} e^{C}$ 


\section{Bogoliubov transformation}

Consider the following Hamiltonian, involving two bosons $R$ and $L$

$$
H=R^{\dagger} R+L^{\dagger} L+\lambda\left(R^{\dagger} L^{\dagger}+L R\right) .
$$

Define two new bosons $S$ and $T$ given by

$$
\begin{aligned}
& S=\alpha R+\beta L^{\dagger}, \\
& T^{\dagger}=\beta R+\alpha L^{\dagger},
\end{aligned}
$$

whose inverse transformation is

$$
\begin{gathered}
R=\alpha S-\beta T^{\dagger}, \\
L^{\dagger}=-\beta S+\alpha T^{\dagger} .
\end{gathered}
$$

For $S$ and $T$ to obey canonical bosonic commutation relations we must have

$$
\left[S, S^{\dagger}\right]=\alpha^{2}-\beta^{2}=1 .
$$

Assuming $\alpha$ and $\beta$ to be real, they can be parametrized by a number $\gamma \in \mathbb{R}$

$$
\begin{gathered}
\alpha=\cosh \gamma, \\
\beta=\sinh \gamma .
\end{gathered}
$$

Plugging (373) into (371), we get

$$
\begin{gathered}
H=\left(\alpha^{2}+\beta^{2}-2 \lambda \alpha \beta\right)\left(S^{\dagger} S+T^{\dagger} T\right) \\
+\left[\lambda\left(\alpha^{2}+\beta^{2}\right)-2 \alpha \beta\right]\left(S^{\dagger} T^{\dagger}+T R\right)+2 \beta(\beta-\alpha \lambda) .
\end{gathered}
$$

By choosing

$$
\tanh 2 \gamma=\lambda,
$$

we can eliminate the crossed terms of Eq. (375) to get (we also drop the constant term)

$$
H=\frac{1}{\cosh 2 \gamma}\left(S^{\dagger} S+T^{\dagger} T\right) .
$$

We can solve explicitly for $\gamma$ in terms of $\lambda$

$$
\gamma=\frac{1}{4} \ln \left(\frac{1+\lambda}{1-\lambda}\right)
$$

Note the condition that $|\lambda|<1$. For later use, we also quote

$$
\begin{aligned}
\cosh 2 \gamma & =\frac{1}{\sqrt{1-\lambda^{2}}} \\
\sinh 2 \gamma & =\frac{\lambda}{\sqrt{1-\lambda^{2}}}, \\
\cosh \gamma & =\frac{1}{2}\left[\left(\frac{1+\lambda}{1-\lambda}\right)^{1 / 4}+\left(\frac{1-\lambda}{1+\lambda}\right)^{1 / 4}\right] \\
\sinh \gamma & =\frac{1}{2}\left[\left(\frac{1+\lambda}{1-\lambda}\right)^{1 / 4}-\left(\frac{1-\lambda}{1+\lambda}\right)^{1 / 4}\right]
\end{aligned}
$$

We thus get

$$
H=\sqrt{1-\lambda^{2}}\left(S^{\dagger} S+T^{\dagger} T\right) .
$$

The spectrum of (383) is now trivial since $S^{\dagger} S$ and $T^{\dagger} T$ are the number operators.

The transformation (373) can also be obtained canonically. The corresponding unitary operator is

$$
\begin{aligned}
U & =\exp \left[\gamma\left(R^{\dagger} L^{\dagger}-L R\right)\right], \\
U^{-1} & =U^{\dagger}
\end{aligned}
$$

such that

$$
\begin{gathered}
U R U^{-1}=\alpha R-\beta L^{\dagger}, \\
U L^{\dagger} U^{-1}=-\beta R+\alpha L^{\dagger} .
\end{gathered}
$$

The above formula can derived by means of the BakerHausdorff formula of Appendix C. The transformed operators should now be read as $R, L \rightarrow S, T$ in order to make contact with (373).

\section{E Single-particle Green's function}

In this Appendix, we calculate the single-particle Green's function for spin- $\frac{1}{2}$ fermions. Since it is diagonal both in the branch ("chirality") and spin indices we need to find

$$
\begin{aligned}
\psi_{R, L \sigma}(x, t) \psi_{R, L \sigma}^{\dagger}(0,0)= & \frac{1}{2 \pi \alpha}\left(\frac{2 \pi \alpha}{L}\right)^{\frac{1}{4}\left(g_{c}+g_{s}+\frac{1}{g_{c}}+\frac{1}{g_{s}}\right)} \times \\
& \exp \left(-i \sqrt{\pi}\left\{c_{c} \varphi_{1,2 c}^{\dagger}\left(x_{ \pm}^{c}\right)+s_{c} \varphi_{2,1 c}^{\dagger}\left(x_{\mp}^{c}\right)+\sigma\left[c_{s} \varphi_{1,2 s}^{\dagger}\left(x_{ \pm}^{s}\right)+s_{s} \varphi_{2,1 s}^{\dagger}\left(x_{\mp}^{s}\right)\right]\right\}\right) \\
& \exp \left(-i \sqrt{\pi}\left\{c_{c} \varphi_{1,2 c}\left(x_{ \pm}^{c}\right)+s_{c} \varphi_{2,1 c}\left(x_{\mp}^{c}\right)+\sigma\left[c_{s} \varphi_{1,2 s}\left(x_{ \pm}^{s}\right)+s_{s} \varphi_{2,1 s}\left(x_{\mp}^{s}\right)\right]\right\}\right) \\
& \exp \left(i \sqrt{\pi}\left\{c_{c} \varphi_{1,2 c}^{\dagger}(0,0)+s_{c} \varphi_{2,1 c}^{\dagger}(0,0)+\sigma\left[c_{s} \varphi_{1,2 s}^{\dagger}(0,0)+s_{s} \varphi_{2,1 s}^{\dagger}(0,0)\right]\right\}\right) \\
& \exp \left(i \sqrt{\pi}\left\{c_{c} \varphi_{1,2 c}(0,0)+s_{c} \varphi_{2,1 c}(0,0)+\sigma\left[c_{s} \varphi_{1,2 s}(0,0)+s_{s} \varphi_{2,1 s}(0,0)\right]\right\}\right)
\end{aligned}
$$


where we have used (321) and already dropped the phase factors in $\hat{N}_{R, L}$. The time dependence, discussed in Section XIV.2 for the spinless case, is easily generalized for electrons with spin through the new entities $x_{ \pm}^{\lambda}=x \mp u_{\lambda} t(\lambda=c, s)$. We can normal order the exponentials by using identity 2 of Appendix $\mathrm{C} e^{A} e^{B}=e^{B} e^{A} e^{C}$, where $C=[A, B]$ and

$$
\begin{aligned}
C= & \pi\left\{c_{c}^{2}\left[\varphi_{1,2 c}\left(x_{ \pm}^{c}\right), \varphi_{1,2 c}^{\dagger}(0,0)\right]+c_{s}^{2}\left[\varphi_{1,2 s}\left(x_{ \pm}^{s}\right), \varphi_{1,2 s}^{\dagger}(0,0)\right]\right. \\
& \left.+s_{c}^{2}\left[\varphi_{2,1 c}\left(x_{\mp}^{c}\right), \varphi_{2,1 c}^{\dagger}(0,0)\right]+s_{s}^{2}\left[\varphi_{2,1 s}\left(x_{\mp}^{s}\right), \varphi_{2,1 s}^{\dagger}(0,0)\right]\right\} \\
\approx & -\frac{1}{2}\left\{c_{c}^{2} \ln \left[\mp \frac{2 \pi i}{L} x_{ \pm}^{c}\right]+c_{s}^{2} \ln \left[\mp \frac{2 \pi i}{L} x_{ \pm}^{s}\right]+s_{c}^{2} \ln \left[ \pm \frac{2 \pi i}{L} x_{\mp}^{c}\right]+s_{s}^{2} \ln \left[ \pm \frac{2 \pi i}{L} x_{\mp}^{s}\right]\right\},
\end{aligned}
$$

where we used (205) and (206) generalized to the spinful case and the limits $L \rightarrow \infty$ and $\alpha \rightarrow 0^{+}$have already been taken. The expectation value of Eq. (387) can now be easily calculated since the expectation value of the normal-ordered sequence of exponentials is equal to 1

$$
\left\langle\psi_{R, L \sigma}(x, t) \psi_{R, L \sigma}^{\dagger}(0,0)\right\rangle_{0}=\frac{1}{2 \pi \alpha}\left(\frac{2 \pi \alpha}{L}\right)^{\frac{1}{4}\left(g_{c}+g_{s}+\frac{1}{g_{c}}+\frac{1}{g_{s}}\right)}\left(\frac{ \pm i L}{2 \pi x_{ \pm}^{c}}\right)^{\frac{c_{c}^{2}}{2}}\left(\frac{ \pm i L}{2 \pi x_{ \pm}^{s}}\right)^{\frac{c_{s}^{2}}{2}}\left(\frac{L}{ \pm i 2 \pi x_{\mp}^{c}}\right)^{\frac{s_{c}^{2}}{2}}\left(\frac{L}{ \pm i 2 \pi x_{\mp}^{s}}\right)^{\frac{s_{s}^{2}}{2}} .
$$

Using

$$
\begin{aligned}
c_{\lambda} & =\frac{1}{2}\left(\frac{1}{\sqrt{g_{\lambda}}}+\sqrt{g_{\lambda}}\right), \\
s_{\lambda} & =\frac{1}{2}\left(\frac{1}{\sqrt{g_{\lambda}}}-\sqrt{g_{\lambda}}\right), \\
c_{\lambda}^{2}+s_{\lambda}^{2} & =\frac{1}{2}\left(\frac{1}{g_{\lambda}}+g_{\lambda}\right),
\end{aligned}
$$

and $c_{\lambda}^{2}=1+s_{\lambda}^{2}$, we have

$$
\begin{aligned}
\left\langle\psi_{R, L \sigma}(x, t) \psi_{R, L \sigma}^{\dagger}(0,0)\right\rangle_{0} & =\frac{ \pm i}{2 \pi \alpha}\left(\frac{\alpha}{x_{ \pm}^{c}}\right)^{\frac{c_{c}^{2}}{2}}\left(\frac{\alpha}{x_{ \pm}^{s}}\right)^{\frac{c_{s}^{2}}{2}}\left(\frac{\alpha}{x_{\mp}^{c}}\right)^{\frac{s_{c}^{2}}{2}}\left(\frac{\alpha}{x_{\mp}^{s}}\right)^{\frac{s_{s}^{2}}{2}} \\
& =\frac{ \pm i}{2 \pi} \frac{1}{\sqrt{x_{ \pm}^{c} x_{ \pm}^{s}}}\left(\frac{\alpha^{2}}{x^{2}-u_{c}^{2} t^{2}}\right)^{\frac{s_{c}^{2}}{2}}\left(\frac{\alpha^{2}}{x^{2}-u_{s}^{2} t^{2}}\right)^{\frac{s_{s}^{2}}{2}} \\
& =\frac{ \pm i}{2 \pi} \frac{1}{\sqrt{x_{ \pm}^{c} x_{ \pm}^{s}}}\left(\frac{\alpha^{2}}{x^{2}-u_{c}^{2} t^{2}}\right)^{\frac{1}{8}\left(\frac{1}{g_{c}}+g_{c}-2\right)}\left(\frac{\alpha^{2}}{x^{2}-u_{s}^{2} t^{2}}\right)^{\frac{1}{8}\left(\frac{1}{g_{s}}+g_{s}-2\right)} .
\end{aligned}
$$

Analogously,

$$
\left\langle\psi_{R, L \sigma}^{\dagger}(0,0) \psi_{R, L \sigma}(x, t)\right\rangle_{0}=\frac{ \pm i}{2 \pi} \frac{1}{\sqrt{x_{ \pm}^{c} x_{ \pm}^{s}}}\left(\frac{\alpha^{2}}{x^{2}-u_{c}^{2} t^{2}}\right)^{\frac{1}{8}\left(\frac{1}{g_{c}}+g_{c}-2\right)}\left(\frac{\alpha^{2}}{x^{2}-u_{s}^{2} t^{2}}\right)^{\frac{1}{8}\left(\frac{1}{g_{s}}+g_{s}-2\right)} .
$$

Thus,

$$
G_{R, L \sigma}(x, t)=\frac{ \pm \operatorname{sgn}(t)}{2 \pi} \frac{1}{\sqrt{\left(x \mp u_{c} t\right)\left(x \mp u_{s} t\right)}}\left(\frac{\alpha^{2}}{x^{2}-u_{c}^{2} t^{2}}\right)^{\frac{1}{8}\left(\frac{1}{g_{c}}+g_{c}-2\right)}\left(\frac{\alpha^{2}}{x^{2}-u_{s}^{2} t^{2}}\right)^{\frac{1}{8}\left(\frac{1}{g_{s}}+g_{s}-2\right)},
$$

and

$$
\tilde{G}_{\sigma}(x, t)=\frac{\operatorname{sgn}(t)}{2 \pi}\left[\frac{e^{i k_{F} x}}{\sqrt{\left(x-u_{c} t\right)\left(x-u_{s} t\right)}}-\frac{e^{-i k_{F} x}}{\sqrt{\left(x+u_{c} t\right)\left(x+u_{s} t\right)}}\right]\left(\frac{\alpha^{2}}{x^{2}-u_{c}^{2} t^{2}}\right)^{\frac{1}{8}\left(g_{c}+\frac{1}{g_{c}}-2\right)}\left(\frac{\alpha^{2}}{x^{2}-u_{s}^{2} t^{2}}\right)^{\frac{1}{8}\left(g_{s}+\frac{1}{g_{s}}-2\right)} .
$$

The spinless case simplifies to

$$
\tilde{G}(x, t)=\frac{\operatorname{sgn}(t)}{2 \pi}\left[\frac{e^{i k_{F} x}}{(x-u t)}-\frac{e^{-i k_{F} x}}{(x+u t)}\right]\left(\frac{\alpha^{2}}{x^{2}-u^{2} t^{2}}\right)^{\frac{1}{4}\left(g+\frac{1}{g}-2\right)} .
$$




\section{References}

[1] O. M. Auslaender, A. Yacoby, R. de Picciotto, K. W. Baldwin, L. N. Pfeiffer, and K. W. West, Phys. Rev. Lett. 84, 1764 (2000).

[2] M. Bockrath, D. H. Cobden, J. Lu, A. G. Rinzler, R. E. Smalley, L. Balents, and P. L. McEuen, Nature 397, 598 (1998).

[3] M. Grayson, D. C. Tsui, L. N. Pfeiffer, K. W. West, and A. M. Chang, Phys. Rev. Lett. 80, 1062 (1998).

[4] V. J. Emery, in Highly Conducting One-Dimensional Solids, edited by J. T. Devreese, R. P. Evrard, and V. E. van Doren (Plenum), New York, 1979), chap. 6, pp. 247-303.

[5] J. Sólyom, Adv. Phys. 28, 201 (1979)

[6] I. Affleck, in Fields, Strings and Critical Phenomena, edited by E. Brézin and J. Zinn-Justin (North-Holland, Amsterdam, 1988), pp. 563-640.

[7] J. Voit, Rep. Prog. Phys. 57, 977 (1994).

[8] J. von Delft and H. Schoeller, Ann. Phys. (Leipzig) 7, 225 (1998), cond-mat/9805275v3.

[9] J. M. Luttinger, J. Math. Phys. 4, 1154 (1963).

[10] D. C. Mattis and E. H. Lieb, J. Math. Phys. 6, 304 (1965).

[11] A. Luther and I. Peschel, Phys. Rev. B 9, 2911 (1974).

[12] A. Luther and I. Peschel, Phys. Rev. B 12, 3908 (1975).
[13] D. C. Mattis, J. Math. Phys. 15, 609 (1974).

[14] R. Heidenreich, R. Seiler, and A. Uhlenbrock, J. Stat. Phys. 22, 27 (1980).

[15] F. D. M. Haldane, J. Phys. C 14, 2585 (1981).

[16] F. D. M. Haldane, Phys. Rev. Lett. 45, 1358 (1980).

[17] J. Hubbard, Proc. R. Soc. A 240, 539 (1957).

[18] J. Hubbard, Proc. R. Soc. A 243, 336 (1958).

[19] J. Hubbard, Proc. R. Soc. A 276, 238 (1963).

[20] E. H. Lieb and F. Y. Wu, Phys. Rev. Lett. 20, 1445 (1968).

[21] C. N. Yang and C. P. Yang, Phys. Rev. 147, 303 (1966).

[22] S. Coleman, Phys. Rev. D 11, 2088 (1975).

[23] S. Mandelstam, Phys. Rev. D 11, 3026 (1975).

[24] P. Jordan and E. Wigner, Z. Phys. 47, 631 (1928).

[25] L. D. Landau, Sov. Physics JETP 3, 920 (1957a).

[26] L. D. Landau, Sov. Phys. JETP 5, 101 (1957b).

[27] L. D. Landau, Sov. Phys. JETP 8, 70 (1959).

[28] H. J. Schulz, Int. J. Mod. Phys. B 5, 57 (1981).

[29] J. Voit, J. Phys. C: Solid State Phys. 21, L1141 (1988).

[30] T. Giamarchi and H. J. Schulz, Phys. Rev. B 39, 4620 (1989).

[31] A. Luther and V. J. Emery, Phys. Rev. Lett. 33, 589 (1974). 